OCHA-PP-305, KEK-TH-1396, UT-HET 041, IC/2010/076

\title{
Higgs boson pair production in new physics models at hadron, lepton, and photon colliders
}

\author{
Eri Asakawa,,$*$ Daisuke Harada, 2,3 , † Shinya Kanemura,, , \\ Yasuhiro Okada, $2,3, \S$ and Koji Tsumura, \\ ${ }^{1}$ Department of Physics, Ochanomizu University, Tokyo 112-8610, Japan \\ ${ }^{2}$ KEK Theory Center, Institute of Particle and Nuclear Studies, \\ KEK, 1-1 Oho, Tsukuba, Ibaraki 305-0801, Japan \\ ${ }^{3}$ Department of Particle and Nuclear Physics, \\ The Graduate University for Advanced Studies (Sokendai), \\ 1-1 Oho, Tsukuba, Ibaraki 305-0801, Japan \\ ${ }^{4}$ Department of Physics, The University of Toyama, \\ 3190 Gofuku, Toyama 930-8555, Japan \\ ${ }^{5}$ The Abdus Salam ICTP of UNESCO and IAEA, \\ Strada Costiera 11, 34151 Trieste, Italy
}

\begin{abstract}
We study Higgs boson pair production processes at future hadron and lepton colliders including the photon collision option in several new physics models; i.e., the two-Higgs-doublet model, the scalar leptoquark model, the sequential fourth generation fermion model and the vectorlike quark model. Cross sections for these processes can deviate significantly from the standard model predictions due to the one-loop correction to the triple Higgs boson coupling constant. For the one-loop induced processes such as $g g \rightarrow h h$ and $\gamma \gamma \rightarrow h h$, where $h$ is the (lightest) Higgs boson and $g$ and $\gamma$ respectively represent a gluon and a photon, the cross sections can also be affected by new physics particles via additional one-loop diagrams. In the two-Higgs-doublet model and scalar leptoquark models, cross sections of $e^{+} e^{-} \rightarrow h h Z$ and $\gamma \gamma \rightarrow h h$ can be enhanced due to the nondecoupling effect in the one-loop corrections to the triple Higgs boson coupling constant. In the sequential fourth generation fermion model, the cross section for $g g \rightarrow h h$ becomes very large because of the loop effect of the fermions. In the vectorlike quark model, effects are small because the theory has decoupling property. Measurements of the Higgs boson pair production processes can be useful to explore new physics through the determination of the Higgs potential.
\end{abstract}

PACS numbers: 12.60.Fr, 14.80.Bn

\footnotetext{
*Electronic address: eri@post.kek.jp

${ }^{\dagger}$ Electronic address: dharada@post.kek.jp

${ }^{\ddagger}$ Electronic address: kanemu@sci.u-toyama.ac.jp

$\S$ Electronic address: yasuhiro.okada@kek.jp

『Electronic address: ktsumura@ictp.it
} 


\section{INTRODUCTION}

The standard model (SM) for particle physics has experienced a great success in describing the experimental data of high energy physics below the energy range of a hundred GeV, but its portion for electroweak symmetry breaking, the Higgs sector, remains unknown. Experimental confirmation of the Higgs boson is one of the most important issues in the high energy physics. The direct search results at the LEP experiment have constrained the mass $\left(m_{h}\right)$ of the Higgs boson as $m_{h} \gtrsim 114.4 \mathrm{GeV}$ [1] in the SM with one Higgs doublet, and the global analysis of precision measurements for electroweak observables has indicated that $m_{h}$ is smaller than $157 \mathrm{GeV}$ at the $95 \%$ confidence level [1, 2]. In addition, the combined data from the CDF and D0 collaborations at the Fermilab Tevatron have excluded the region of $162 \mathrm{GeV} \lesssim m_{h} \lesssim 166 \mathrm{GeV}[3]^{1}$. The CERN Large Hadron Collider (LHC) has already started its operation, and it will soon be ready for hunting the Higgs boson. We expect that the Higgs boson will be discovered in coming several years.

Once the Higgs boson is found at the Tevatron or the LHC, its property such as the mass, the decay width, production cross sections and the decay branching ratios will be thoroughly measured as accurately as possible in order to confirm whether it is really the particle responsible for spontaneous breaking of the electroweak symmetry. The Higgs mechanism will be tested by determining the coupling constants of the Higgs boson to the weak gauge bosons. The measurement of the Yukawa coupling constants will clarify the mass generation mechanism of quarks and charged leptons. However, in order to understand the physics behind the electroweak symmetry breaking, the Higgs potential must be reconstructed by measuring the triple Higgs boson coupling constant (the hhh coupling constant).

On the other hand, from the theoretical view point, it would be expected that the SM is replaced by a more fundamental theory at the $\mathrm{TeV}$ scale. One way to see the new dynamics is to measure effective vertices of the SM fields and to compare them to the theoretical calculation of radiative corrections. The effect can be significant in the electroweak theory especially when the mass of a new particle comes mainly from the vacuum expectation value (VEV) of the Higgs field like chiral fermions. In such a case the decoupling theorem [5] does not necessarily hold, so that the new physics effects do not decouple and are significant. It is well known that the systematic study of nondecoupling parameters in radiative corrections to the gauge boson two point functions has played an important role to constrain new physics models by using the precision data of electroweak observables at the LEP and the Stanford Linear Collider (SLC) [6].

Such nondecoupling effects of new physics particles can also be very significant in the radiative corrections to the $h$ hh coupling constant [7]. Quartic powerlike contributions of the mass of a new particle can appear in the one-loop correction to the $h h h$ coupling constant, which can give a large deviation from the SM prediction. For example, in the twoHiggs-doublet model (THDM), the $h$ hh coupling constant of the lightest (SM-like) Higgs

\footnotetext{
1 Recently, the bound on the Higgs boson mass from Tevatron experiments has been updated[4].
} 
boson can be deviated by $\mathcal{O}(100) \%$ due to nondecoupling effects of extra scalar bosons in radiative corrections without contradiction with perturbative unitarity [7]. It is known that such a large deviation in the $h h h$ coupling constant from the SM value can be a common feature of the Higgs sector with the strong first order electroweak phase transition [8-12], which is required for a successful scenario of electroweak baryogenesis [13]. Therefore, the measurement of the $h h h$ coupling constant at collider experiments can be an important probe into such a cosmological scenario. The one-loop contributions to the $h h h$ coupling constant can also be very large in the model with sequential fourth generation fermions [14] and a class of extended supersymmetric SMs [15].

At the LHC, the measurement of the $h$ hh coupling constant would be challenging. In the SM, the cross section of double Higgs boson production from gluon fusion, $g g \rightarrow h h\lfloor 16$ 18], can be $\mathcal{O}(10) \mathrm{fb}$ for $m_{h}=120-160 \mathrm{GeV}$ with the collision energy of $\sqrt{s}=14 \mathrm{TeV}$, while those of double-Higgs-strahlung $q \bar{q} \rightarrow V^{*} \rightarrow h h V$ [18, 19] and vector boson fusion $q \bar{q} \rightarrow V^{*} V^{*} q \bar{q} \rightarrow h h q \bar{q}$ [16, 18, 20] are much smaller. The double Higgs boson production mechanism from gluon fusion has been studied in Ref. [21] with the $h \rightarrow W W^{(*)}$ decay mode. They conclude that the luminosity of $3000 \mathrm{fb}^{-1}$ is required to measure the $h h h$ coupling constant at the $20-30 \%$ level [21]. For a light Higgs boson $\left(m_{h} \lesssim 130 \mathrm{GeV}\right)$, the main decay mode is $h \rightarrow b \bar{b}$ which cannot be useful due to huge QCD backgrounds, so that the $h h h$ coupling constant cannot be accurately measured at the LHC.

At the International Linear Collider (ILC), the accuracy for measuring the $h h h$ coupling constant would be better than that at the LHC depending on the mass of the Higgs boson. A Higgs boson pair can be produced in the double-Higgs-strahlung process $e^{+} e^{-} \rightarrow h h Z$ [22] and the $W$ fusion mechanism $e^{+} e^{-} \rightarrow h h \nu \bar{\nu}$ [23]. At the first stage of the ILC where $e^{+} e^{-}$ energy is $500 \mathrm{GeV}$ the $h h h$ coupling can be measured via the double-Higgs-strahlung process for $m_{h} \lesssim 140 \mathrm{GeV}$ [24 27]. The evaluation of the statistical sensitivity for the $h h h$ coupling constant is about 20\% accuracy [24, 25]. Detailed simulation studies for this process are ongoing, which shows that the sensitivity may be lower [28]. The photon linear collider (PLC) option may also be useful to explore the hhh coupling constant for $120 \lesssim m_{h} \lesssim 200$ $\mathrm{GeV}$ [29, 30]. A simulation study is also in progress [31]. At the second stage of the ILC $(\sqrt{s}=1 \mathrm{TeV})$ or the Compact Linear Collider (CLIC) where the collision energy would be at a multi-TeV scale, the double Higgs boson pair production from $W$ boson fusion becomes important because the cross section is larger due to the $t$-channel enhancement [25, 27]. The statistical sensitivity for the $h h h$ coupling constant is less than $10 \%$ [25].

In this paper, we study how the $h$ hh coupling constant affects cross sections for the double Higgs boson production processes $g g \rightarrow h h, e^{+} e^{-} \rightarrow h h Z, e^{+} e^{-} \rightarrow h h \nu \bar{\nu}$ and $\gamma \gamma \rightarrow h h$ in various new physics models such as the THDM, models with scalar leptoquarks, the model with the chiral fourth generation fermions and the model with vectorlike quarks. Cross sections for these Higgs boson pair production processes are evaluated, and can deviate significantly from the SM predictions due to the deviation in the one-loop corrected $h h h$ coupling constant. In these processes, the effect of the deviation in the $h h h$ coupling constant mainly appears in the interference of the diagram with the $h h h$ coupling constant and the other diagrams. Thus, the sign of the deviation can be important. Also, in the one-loop 
induced processes such as $g g \rightarrow h h$ and $\gamma \gamma \rightarrow h h$, cross sections can depend on new physics particles in additional one-loop diagrams. In the THDM and scalar leptoquark models, cross sections for $e^{+} e^{-} \rightarrow h h Z$ and $\gamma \gamma \rightarrow h h$ can be enhanced due to the nondecoupling effect on the $h h h$ coupling constant through the extra scalar loops. In the chiral fourth generation model, cross sections of double Higgs boson production processes can become significantly large, because new particles mediate in the leading order loop diagram as well as the nondecoupling effect on the $h h h$ coupling constant. In models with vectorlike quarks, the effect on the cross sections are small because of the decoupling nature of the theory. By measuring these double Higgs boson production processes at different future collider experiments, we would be able to test properties of new physics particles in the loop, which helps identify the new physics model.

In Sec. II, effects of the $h h h$ coupling constant in Higgs boson pair production processes $g g \rightarrow h h$ at LHC, $e^{+} e^{-} \rightarrow h h Z$ and $e^{+} e^{-} \rightarrow h h \nu \bar{\nu}$ at ILC and CLIC, and $\gamma \gamma \rightarrow h h$ at their photon collider options are discussed. Model dependent analyses for these processes are given in Sec. III for the THDM, the scalar leptoquark models, the chiral fourth generation model, and the vectorlike quarks. In Sec. IV, summary and discussions are given.

\section{THE HIGGS BOSON PAIR PRODUCTION PROCESSES AT COLLIDERS}

In this section, we discuss Higgs boson pair production processes $g g \rightarrow$ hh [16 18], $e^{+} e^{-} \rightarrow h h Z$ [22], $e^{+} e^{-} \rightarrow h h \nu \bar{\nu}$ [23] and $\gamma \gamma \rightarrow h h$ [29] in various new physics models. These processes contain the $h h h$ coupling constant so that they can be used to determine the $h h h$ coupling constant at future collider experiments. The effective $g g h$ and $\gamma \gamma h$ vertices would be precisely measured in the single Higgs boson production processes as $g g \rightarrow h$ at hadron colliders [32] and $\gamma \gamma \rightarrow h$ resonance production at the PLC [33], which will be used to extract the $h h h$ coupling constant from the one-loop induced processes such as $g g \rightarrow h h$ and $\gamma \gamma \rightarrow h h$. In this section, before going to the discussion on the calculation for the cross sections in each model, we first consider the results in the SM with a constant shift of the $h h h$ coupling constant by a factor of $(1+\Delta \kappa)$;

$$
\lambda_{h h h}=\lambda_{h h h}^{\mathrm{SM}}(1+\Delta \kappa),
$$

where $\lambda_{h h h}^{\mathrm{SM}}=-3 m_{h}^{2} / v$ at the tree level ${ }^{2}$ with $v(\simeq 246 \mathrm{GeV})$ being the VEV and $m_{h}$ being the mass of the Higgs boson $h$. This constant shift can be realized when there is

${ }^{2}$ At the one-loop order, the effective $h h h$ vertex function have been evaluated as [7]

$$
\Gamma_{h h h}^{\mathrm{SM}}\left(\hat{s}, m_{h}^{2}, m_{h}^{2}\right) \simeq-\frac{3 m_{h}^{2}}{v}\left\{1-\frac{N_{c} m_{t}^{4}}{3 \pi^{2} v^{2} m_{h}^{2}}\left[1+\mathcal{O}\left(\frac{m_{h}^{2}}{m_{t}^{2}}, \frac{\hat{s}}{m_{t}^{2}}\right)\right]\right\}
$$

where $N_{c}(=3)$ is the color factor. The full expression of the vertex function $\Gamma_{h h h}^{\mathrm{SM}}\left(p_{1}^{2}, p_{2}^{2}, p_{3}^{2}\right)$ is also given in Appendix A for completeness. In numerical analysis, we include the SM one-loop correction to the $h h h$ coupling constant. 


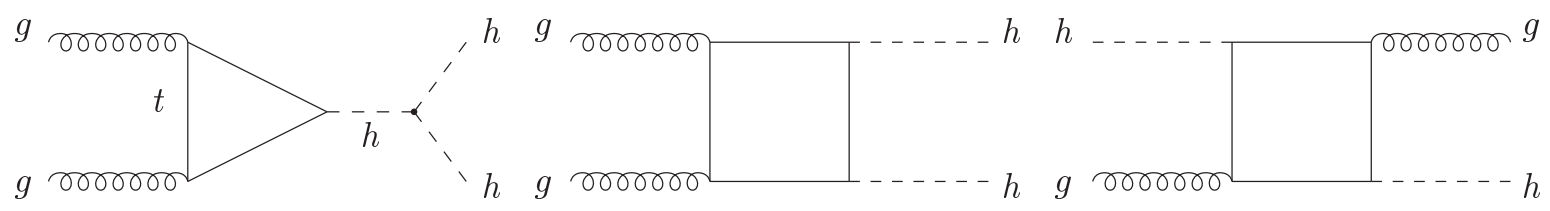

FIG. 1: The double Higgs boson production process $g g \rightarrow h h$ via gluon fusion at the hadron collider.

the dimension six operator in the Higgs potential [29, 34]. Quantum corrections to the $h h h$ coupling constant due to the bosonic loop can also provide the constant shift of the hhh coupling constant approximately [7].

At the LHC, the largest cross section of the Higgs boson pair production comes from the gluon fusion mechanism [16 18]. Feynman diagrams for $g g \rightarrow h h$ are depicted in FIG. 1, The triangular loop diagrams contain information of the $h h h$ coupling constant. The parton level cross sections are calculated at the leading order as [17]

$$
\widehat{\sigma}(g g \rightarrow h h)=\int_{\hat{t}_{-}}^{\hat{t}_{+}} d \hat{t} \frac{1}{2^{2}} \frac{1}{8^{2}} \frac{1}{2 !} \frac{1}{16 \pi \hat{s}^{2}} \frac{2 \alpha_{S}^{2}}{(4 \pi)^{2}}\left\{\left|\frac{\lambda_{h h h} v}{\hat{s}-m_{h}^{2}} F_{\triangle}+F_{\square}\right|^{2}+\left|G_{\square}\right|^{2}\right\},
$$

where $F_{\triangle}$ is the loop function for the triangular diagram, while $F_{\square}$ and $G_{\square}$ are those for box diagrams which, respectively, correspond to the invariant amplitudes for same and opposite polarizations of incoming gluons [21]: see Appendix B. The invariant mass distribution can be obtained by multiplying the gluon-gluon luminosity function as

$$
\frac{d \sigma(g g \rightarrow h h)}{d M_{h h}}=\frac{2 M_{h h}}{s} \widehat{\sigma}(g g \rightarrow h h) \frac{d L_{g g}}{d \tau},
$$

where $M_{h h}=\sqrt{\hat{s}}, \tau=\hat{s} / s$, and

$$
\frac{d L_{g g}}{d \tau}=\int_{\tau}^{1} \frac{d x}{x} f_{g}\left(x, \mu_{F}=M_{h h}\right) f_{g}\left(\tau / x, \mu_{F}=M_{h h}\right),
$$

where $f_{g}\left(x, \mu_{F}\right)$ is the parton distribution function of gluons. In our numerical calculation, the CTEQ6L parton distribution function is used [35]. The loop integrals are evaluated by a package; LoopTools [36].

It is well known that this process receives large QCD corrections ${ }^{3}$. Although the NLO calculation is very important in evaluating this process, throughout this paper we totally

3 The next-to-leading order (NLO) QCD corrections to this process have been computed in the heavy top-quark mass limit in Ref. [37], which give an over all factor $K \simeq 1.9$ (K-factor) for $\mu_{F}=M_{h h}$. The smaller value of $K \simeq 1.65$ for $\mu_{F}=m_{h}$ was suggested by Ref. [21]. The correction mainly comes from the initial state radiation of gluons. It is known that this kind of approximation works well in the single Higgs production via the gluon fusion mechanism, where the NLO cross section is evaluated by the leading order $g g \rightarrow h$ cross section for a finite top-quark mass with the $K$-factor in the large $m_{t}$ limit. The running of strong coupling constant can also change the cross section by $25-50 \%$ [21]. 

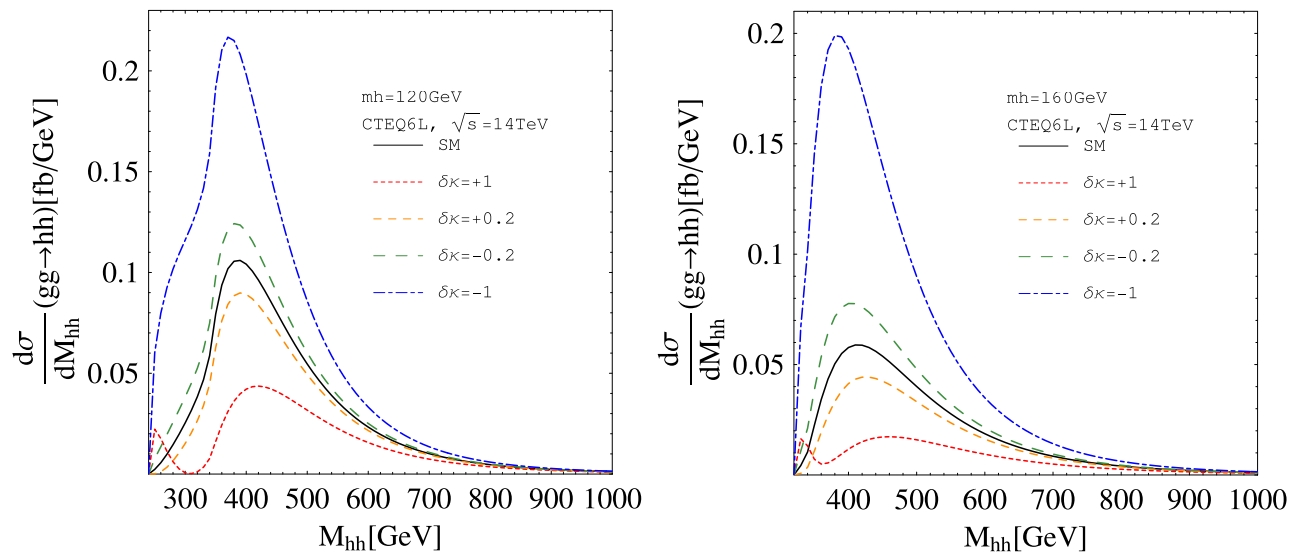

FIG. 2: The invariant mass distribution of the cross section of $g g \rightarrow h h$ process at the LHC with $\sqrt{s}=14 \mathrm{TeV}$ for $m_{h}=120 \mathrm{GeV}$ (left) and $m_{h}=160 \mathrm{GeV}$ (right). The solid, dotted, dashed, long-dashed and dot-dashed curved lines denote the SM prediction, the SM with the positive 100\% correction to the $h h h$ coupling constant, that with the $+20 \%$ correction, that with the $-20 \%$ correction, and that with the $-100 \%$ correction, respectively.

neglect NLO QCD corrections in our calculations of the cross section in various new physics models. The QCD corrections in each new physics model are currently unknown so that the computation of these corrections is beyond the scope of this paper.

In FIG. 2, we show the invariant mass distributions of the cross section of $g g \rightarrow h h$ process with the deviation of the $h h h$ coupling constant for $m_{h}=120 \mathrm{GeV}$ (left) and for $m_{h}=160 \mathrm{GeV}$ (right). Throughout this paper we take the top-quark mass to be 171.2 GeV. These solid, dotted/dashed and long-dashed/dot-dashed curves represent the SM prediction including the SM one-loop effect on the hhh coupling constant, that with constructive deviations $\Delta \kappa=+1.0$ and +0.2 and that with destructive deviations $\Delta \kappa=-1.0$ and -0.2 , respectively. The total cross section is about 20 (10) fb for $m_{h}=120$ (160) GeV in the SM. Only for $\Delta \kappa=+1.0$, a small peak comes from the large $h h h$ coupling constant through the triangular diagram in the near threshold region. The peaks can be found around $M_{h h} \sim 400 \mathrm{GeV}$, which are caused by the interference effect of the triangular and the box diagrams. Since these two contributions are destructive to each other, the positive (negative) variation of the $h$ hh coupling constant makes the cross sections small (large) in this process. This means that in the $g g \rightarrow h h$ process the sensitivity is getting better for the negative contribution to the $h h h$ coupling constant and vice versa. If we have additional colored particles in the new physics model, this situation could be changed.

At an electron-positron linear collider, the hhh coupling constant will be measured by the double-Higgs-strahlung [22] and the Higgs boson pair production via the $W$ boson fusion mechanism [23]. Feynman diagrams for these processes are shown in FIG. 3. The $e^{+} e^{-} \rightarrow$ $h h Z$ process may be a promising channel at the ILC to measure the $h h h$ coupling constant for light Higgs bosons because of the simple kinematical structure. Since relatively larger collision energy is required for three body final states of $h h Z$, the $s$-channel nature of the 


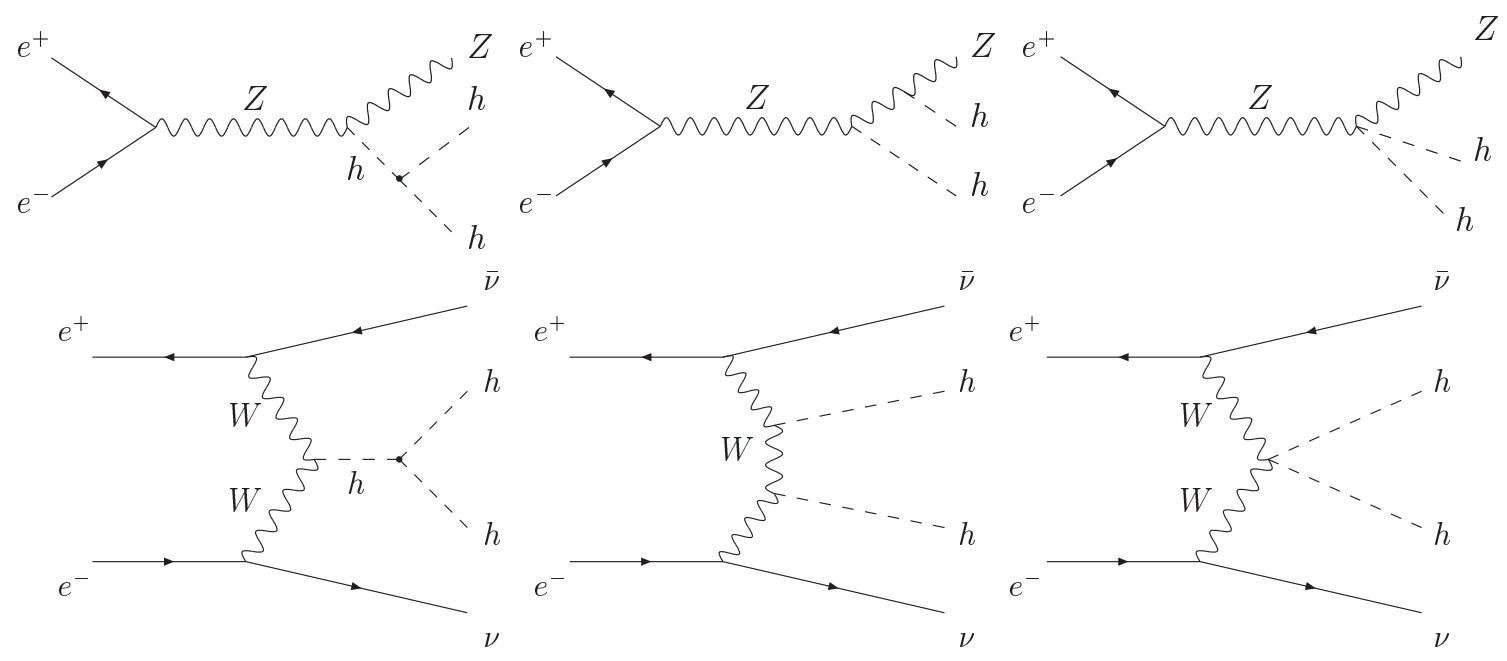

FIG. 3: The double Higgs boson production at the $e^{+} e^{-}$collider. The double-Higgs-strahlung process $e^{+} e^{-} \rightarrow h h Z$ and the vector boson fusion process $e^{+} e^{-} \rightarrow h h \nu_{e} \bar{\nu}_{e}$.
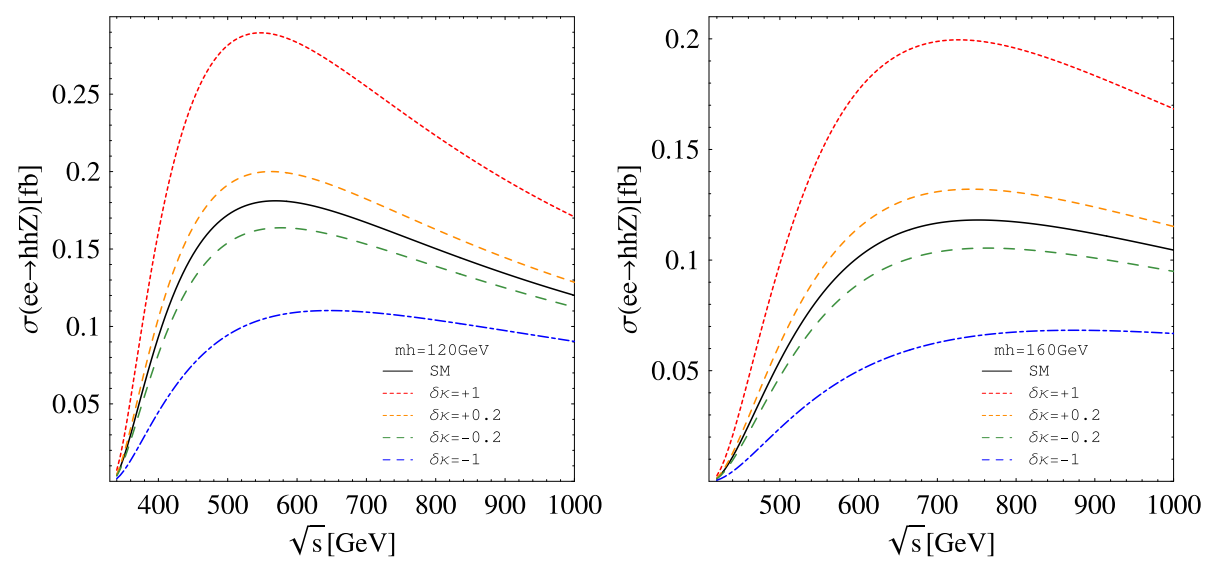

FIG. 4: The cross sections of $e^{+} e^{-} \rightarrow h h Z$ process at the ILC as a function of collision energy $\sqrt{s}$ for $m_{h}=120 \mathrm{GeV}$ (left) and $m_{h}=160 \mathrm{GeV}$ (right).

process may decrease the cross section. On the other hand, if we have large enough energy, one can control the collision energy to obtain the maximal production rate. In FIG. 4, the cross sections of the double-Higgs-strahlung are evaluated as a function of $e^{+} e^{-}$center of mass energy $\sqrt{s}$. The left (right) panel shows the case with the Higgs boson mass to be $m_{h}=120(160) \mathrm{GeV}$. The curves are presented in the same manner as in FIG. 2. Under the variation of the $h h h$ coupling constant, the cross section of the double-Higgs-strahlung has the opposite correlation to that of $g g \rightarrow h h$. Therefore, the positive contributions to the hhh coupling constant has an advantage to obtain better sensitivities.

At a high energy lepton collider, the hard photons can be obtained from the Compton back scattering method [38]. By using hard photons, Higgs boson pairs can be produced in $\gamma \gamma \rightarrow h$ process. Feynman diagrams for this process are shown in FIG. 5, and the helicity 


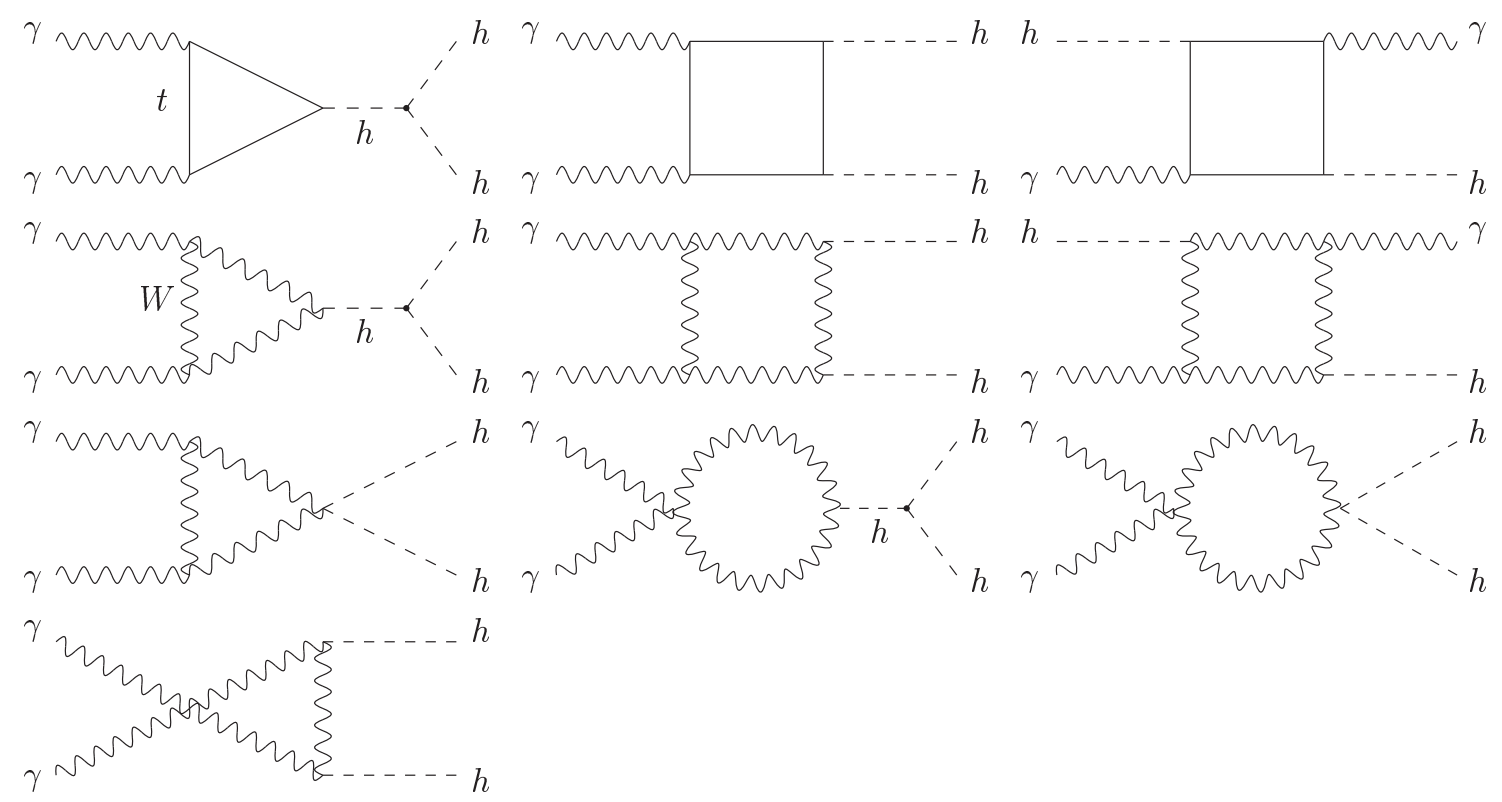

FIG. 5: The double Higgs boson production process $\gamma \gamma \rightarrow h h$ at the photon collider.

specified cross sections are given by

$$
\hat{\sigma}^{\lambda_{1} \lambda_{2}} \equiv \widehat{\sigma}\left(\gamma_{\lambda_{1}} \gamma_{\lambda_{2}} \rightarrow h h\right)=\int_{\hat{t}_{-}}^{\hat{t}_{+}} d \hat{t} \frac{1}{2 !} \frac{1}{16 \pi \hat{s}^{2}} \frac{\alpha_{\mathrm{EM}}^{2}}{(4 \pi)^{2}}\left|\frac{\lambda_{h h h} v}{\hat{s}-m_{h}^{2}} H_{\triangle}^{\lambda_{1} \lambda_{2}}+H_{\square}^{\lambda_{1} \lambda_{2}}\right|^{2},
$$

where $H_{\triangle}^{\lambda_{1} \lambda_{2}}$ and $H_{\square}^{\lambda_{1} \lambda_{2}}$ are the loop functions [29] (see Appendix B). The total cross section is calculated by convoluting with the photon luminosity function $f_{\gamma}(y, x)$, where $x=4 E_{e} \omega_{0} / m_{e}^{2}$ can be controlled by the frequency $\omega_{0}$ of the laser photon, as

$$
\sigma_{\gamma \gamma \rightarrow h h}^{e e}=\int_{\tau_{h h}}^{y_{m}^{2}} d \tau \int_{\tau}^{y_{m}} \frac{d y}{y}\left[\frac{1+\xi_{1}^{\gamma} \xi_{2}^{\gamma}}{2} \hat{\sigma}^{++}+\frac{1-\xi_{1}^{\gamma} \xi_{2}^{\gamma}}{2} \hat{\sigma}^{+-}\right] f_{\gamma}(y, x) f_{\gamma}(\tau / y, x),
$$

where $\xi^{\gamma}$ is the mean helicity of the photon. The photon luminosity spectrum is given by

$$
\begin{aligned}
f_{\gamma}(y, x)= & \frac{1}{D(x)}\left[\frac{1}{1-y}+1-y-4 r(1-r)-2 \lambda^{e} \lambda^{\gamma} r x(2 r-1)(2-y)\right], \\
D(x)= & \left(1-\frac{4}{x}-\frac{8}{x^{2}}\right) \ln (1+x)+\frac{1}{2}+\frac{8}{x}-\frac{1}{2(1+x)^{2}} \\
& +2 \lambda^{e} \lambda^{\gamma}\left[\left(1+\frac{2}{x}\right) \ln (1+x)-\frac{5}{2}+\frac{1}{1+x}-\frac{1}{2(1+x)^{2}}\right],
\end{aligned}
$$

where $r=\frac{y}{x(1-y)}$ and $\lambda^{e}\left(\lambda^{\gamma}\right)$ is the helicity of the incident electron (photon) [38]. The maximal energy fraction of photon $y_{m}=\frac{x}{1+x}$ is fixed by the kinematics of the Compton scattering at the photon collider. In FIG. 6, the full cross sections of $e^{-} e^{-}(\gamma(+) \gamma(+)) \rightarrow h h$ are shown as a function of the energy of the $e^{-} e^{-}$system. We here choose the same sign polarizations for initial photons in order to efficiently extract information of the $h h h$ coupling constant. The parameter $x$ is taken to be 4.8 , which can be tuned by the frequency of the 

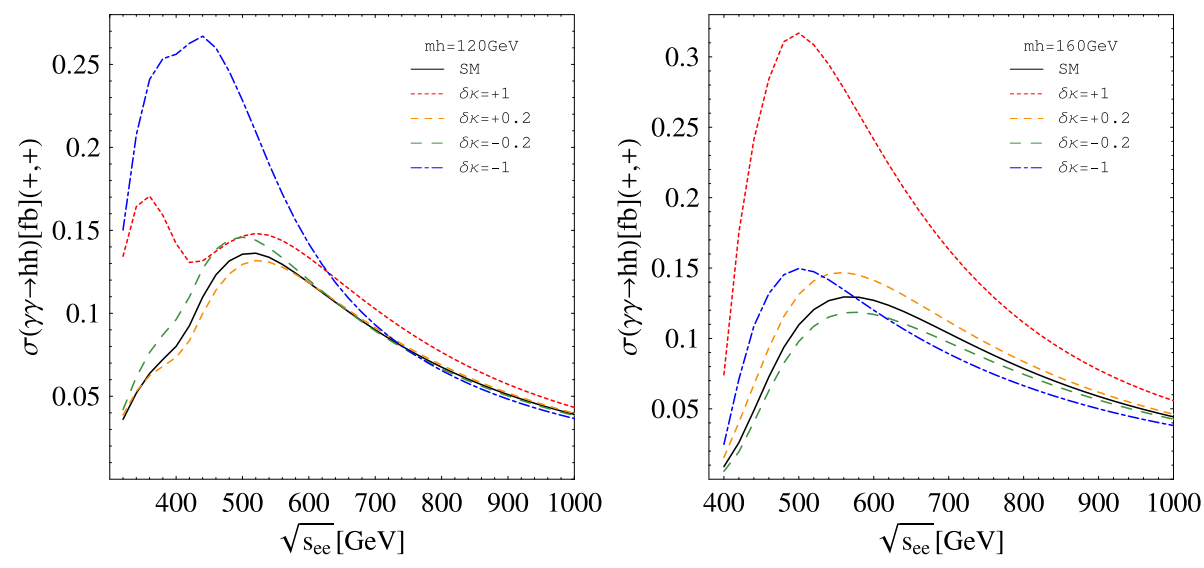

FIG. 6: The full cross section of $e^{-} e^{-}(\gamma(+) \gamma(+)) \rightarrow h h$ process as a function of $\sqrt{s}_{e e}$ for $m_{h}=120$ $\mathrm{GeV}$ (left) and $m_{h}=160 \mathrm{GeV}$ (right).
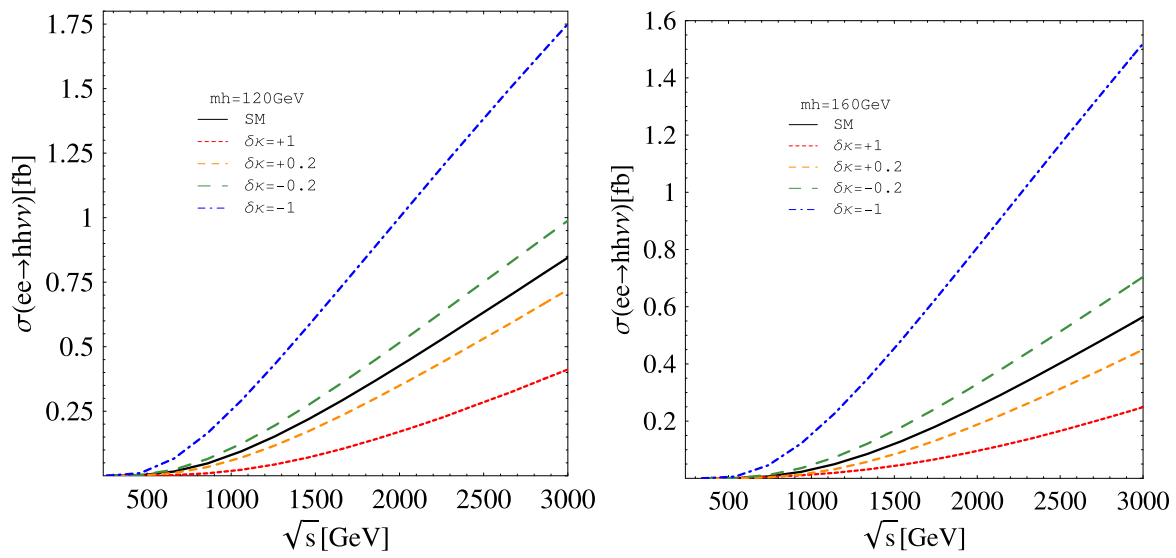

FIG. 7: The cross sections of $e^{+} e^{-} \rightarrow h h \nu \bar{\nu}$ process at the ILC as a function of collision energy $\sqrt{s}$ for $m_{h}=120 \mathrm{GeV}$ (left) and $m_{h}=160 \mathrm{GeV}$ (right).

laser photon. The curves are given in the same manner as in FIG. 2. The situation is very different from $g g \rightarrow h h$ at the LHC. Energies of initial gluons are widely varied at a hadron collider, while back-scattered photons at the PLC have narrow band spectra. Therefore, we can tune the effective energy of photons at the PLC to some extent. The relative strength of the $W$ boson and the top-quark loop diagrams strongly depends on the collision energy and the Higgs boson mass. Only for $m_{h}=120 \mathrm{GeV}$, the large $h h h$ coupling constant case $(\Delta \kappa=+1.0)$ shows a peak at the near threshold regime. It is found that the negative deviation of the $h h h$ coupling constant makes cross section large for $m_{h}=120 \mathrm{GeV}$ (left), while it has an opposite effect on the cross section for $m_{h}=160 \mathrm{GeV}$ (right).

If we go to further high energy $e^{+} e^{-}$colliders, the second stage of the ILC or the CLIC, the Higgs boson pair production via the $W$ boson fusion mechanism becomes important [23]. The cross section increases for higher energy because of the $t$-channel enhancement of $W^{+} W^{-} \rightarrow h h$ subprocess. In FIG. 7, we evaluate the production rate for $e^{+} e^{-} \rightarrow h h \nu \bar{\nu}$ by CalcHEP [39]. For both $m_{h}=120 \mathrm{GeV}$ (left) and $m_{h}=160 \mathrm{GeV}$ (right) cases, the 
cross section simply scales as a function of energy and can be much larger than those of $e^{+} e^{-} \rightarrow h h Z$ and $\gamma \gamma \rightarrow h h$. The $\Delta \kappa$ dependence in the cross section of $e^{+} e^{-} \rightarrow h h \nu \bar{\nu}$ is opposite to that in $e^{+} e^{-} \rightarrow h h Z$; i.e., a larger cross section for $e^{+} e^{-} \rightarrow h h \nu \bar{\nu}$ is obtained for a larger $|\Delta \kappa|$ value with a negative sign.

\section{COMPARISON OF THE HIGGS BOSON PAIR CREATION PROCESSES IN DIFFERENT MODELS}

In this section, we study cross sections for the double Higgs boson production processes $g g \rightarrow h h$ [16 18], $e^{+} e^{-} \rightarrow h h Z$ [22], $e^{+} e^{-} \rightarrow h h \nu \bar{\nu}$ [23] and $\gamma \gamma \rightarrow h h$ [29] in four different models; i.e., the THDM, the model with scalar leptoquarks, that with chiral fourth generation quarks and leptons, and that with vectorlike quarks. In these models, one-loop contributions to the $h$ hh coupling constant can be nondecoupling even when new particles are heavier than the electroweak scale, so that large deviations in the $h h h$ coupling constant can affect the cross sections of these double Higgs boson production processes. Unlike the analysis with the constant shift with $\Delta \kappa$ in the previous section, the energy dependencies in the $h h h$ vertex function are also included in our evaluation here. Furthermore, for the one-loop induced processes such as $g g \rightarrow h h$ and $\gamma \gamma \rightarrow h h$, the contribution of additional one-loop diagrams where the new particles are running in the loop can be significant.

\section{A. Two-Higgs-doublet model}

The THDM [40] is the simplest extension of the Higgs sector in the SM, which can appear in various new physics scenarios such as the minimal supersymmetric SM [41], the top color model [42], radiative seesaw models for neutrinos [11, 43] and models of electroweak baryogenesis [44]. The Higgs scalar doublets interact with other fields purely by the electroweak force. Therefore, in addition to the change in the cross section due to the quantum correction to the $h h h$ coupling constant, the contribution of the charged Higgs boson loop can affect the cross section of $\gamma \gamma \rightarrow h h$, while the loop effect of extra scalar bosons appear only through the correction to the $h h h$ coupling constant for $g g \rightarrow h h, e^{+} e^{-} \rightarrow h h Z$ and $e^{+} e^{-} \rightarrow h h \nu \bar{\nu}$.

The potential of the THDM with a softly-broken discrete $Z_{2}$ symmetry is given by

$$
\begin{aligned}
V_{\mathrm{THDM}}= & m_{1}^{2} \Phi_{1}^{\dagger} \Phi_{1}+m_{2}^{2} \Phi_{2}^{\dagger} \Phi_{2}-\left(m_{3}^{2} \Phi_{1}^{\dagger} \Phi_{2}+\text { H.c. }\right)+\frac{\lambda_{1}}{2}\left(\Phi_{1}^{\dagger} \Phi_{1}\right)^{2}+\frac{\lambda_{2}}{2}\left(\Phi_{2}^{\dagger} \Phi_{2}\right)^{2} \\
& +\lambda_{3}\left(\Phi_{1}^{\dagger} \Phi_{1}\right)\left(\Phi_{2}^{\dagger} \Phi_{2}\right)+\lambda_{4}\left(\Phi_{1}^{\dagger} \Phi_{2}\right)\left(\Phi_{2}^{\dagger} \Phi_{1}\right)+\left[\frac{\lambda_{5}}{2}\left(\Phi_{1}^{\dagger} \Phi_{2}\right)^{2}+\text { H.c. }\right]
\end{aligned}
$$

where $\Phi_{i}(i=1,2)$ are scalar isospin doublet fields with the hypercharge of $+1 / 2$, which transform as $\Phi_{1} \rightarrow \Phi_{1}$ and $\Phi_{2} \rightarrow-\Phi_{2}$ under the $Z_{2}$. Although $m_{3}^{2}$ and $\lambda_{5}$ are complex in general, we here take them to be real assuming the CP invariance. The two Higgs doublet 
fields can then be parameterized as

$$
\Phi_{i}=\left(\begin{array}{c}
\omega_{i}^{+} \\
\frac{1}{\sqrt{2}}\left(v_{i}+h_{i}+i z_{i}\right)
\end{array}\right) .
$$

There are 8 degrees of freedom in the two complex scalar doublet fields. Three of them are absorbed as the longitudinal components of the weak gauge bosons. The remaining five convert into the mass eigenstates, two CP even Higgs bosons $(h, H)$, a $\mathrm{CP}$ odd Higgs boson $(A)$, and a pair of charged Higgs bosons $\left(H^{ \pm}\right)$. The eight parameters $m_{1}^{2}-m_{3}^{2}$ and $\lambda_{1}-\lambda_{5}$ are replaced by the $\mathrm{VEV} v$, the mixing angle of $\mathrm{CP}$ even Higgs bosons $\alpha$, the ratio of VEVs $\tan \beta=v_{2} / v_{1}$, the Higgs boson masses $m_{h}, m_{H}, m_{A}, m_{H^{ \pm}}$and the soft breaking parameter $M^{2}=m_{3}^{2} /(\sin \beta \cos \beta)$. The parameters in the Higgs potential can be constrained by imposing theoretical assumptions such as perturbative unitarity [45, 46] and vacuum stability [47]. If we require stability of the theory below a given cutoff scale $\Lambda$ imposing the conditions of vacuum stability and triviality, the Higgs boson parameters are constrained as a function of $\Lambda$ by the renormalization group equation analysis [48].

Here, we consider the "SM-like" case with $\sin (\beta-\alpha)=1$ where only the lighter CP-even Higgs boson $h$ couples to the weak gauge boson as $V V h$ [49]. In this case, all the coupling constants of $h$ to the SM particles take the same form as those in the SM at the tree level. The hhh coupling constant is also described by the same tree-level formula as in the SM. The difference appears at the loop level due to the one-loop contribution of the extra scalars.

Under the imposed softly-broken discrete symmetry, there can be four types of Yukawa interactions [50, 51]. Although in general there can be large phenomenological differences among the different types of Yukawa interaction, especially in flavor physics, we do not specify the type of Yukawa interaction in this paper, because there is no proper difference in the discussion here in the SM-like limit where $h$ behaves as if it were the SM Higgs boson at the tree level ${ }^{4}$.

The extra Higgs bosons have been searched at the LEP experiment. The lower mass bound for the CP-even Higgs boson is $m_{H}>92.8 \mathrm{GeV}$, and that for the CP-odd Higgs boson is $m_{A}>93.4 \mathrm{GeV}$ in the minimal supersymmetric SM whose Higgs sector is the THDM [1]. The bound for charged Higgs boson mass has also been set as $m_{H^{ \pm}}>79.3 \mathrm{GeV}$ [1]. The electroweak precision data from the LEP experiment may indicate that the Higgs sector approximately respects the custodial $S U(2)$ symmetry [53]. This symmetry becomes exact in the Higgs potential in the limit of $m_{A}=m_{H^{ \pm}}$with arbitrary $\sin (\beta-\alpha)$ or in the limit of $m_{H}=m_{H^{ \pm}}$with $\sin (\beta-\alpha)=1$ (or $m_{h}=m_{H^{ \pm}}$with $\cos (\beta-\alpha)=1$ ). The breaking of the custodial symmetry in the two-Higgs-doublet potential gives large contribution to the $\widehat{T}$ parameter which is proportional to the mass differences of extra Higgs bosons. In order to suppress these contributions, we take their masses to be degenerate in the following discussion; i.e., $m_{H}=m_{A}=m_{H^{ \pm}}$.

\footnotetext{
4 The allowed regions of $m_{H^{ \pm}}$and $\tan \beta$ can receive constraint from the flavor physics data such as $b \rightarrow s \gamma$ depending on the type of Yukawa interaction [50, 52].
} 

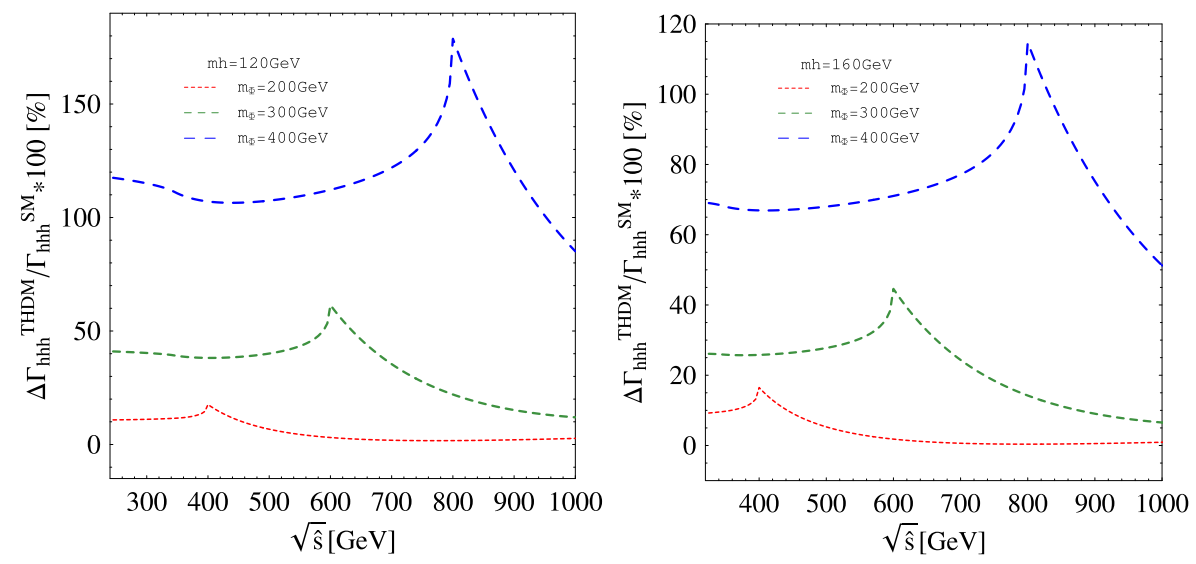

FIG. 8: The rates for one-loop contributions from $H, A, H^{ \pm}$in the THDM to the $h h h$ coupling constant for $m_{h}=120 \mathrm{GeV}$ (left) and for $m_{h}=160 \mathrm{GeV}$ (right).

The one-loop correction to the $h h h$ coupling constant in the THDM is evaluated as [7]

$$
\frac{\Gamma_{h h h}^{\mathrm{THDM}}}{\Gamma_{h h h}^{\mathrm{SM}}} \simeq 1+\frac{m_{H^{ \pm}}^{4}}{6 \pi^{2} v^{2} m_{h}^{2}}\left(1-\frac{M^{2}}{m_{H^{ \pm}}^{2}}\right)^{3}+\frac{m_{H}^{4}}{12 \pi^{2} v^{2} m_{h}^{2}}\left(1-\frac{M^{2}}{m_{H}^{2}}\right)^{3}+\frac{m_{A}^{4}}{12 \pi^{2} v^{2} m_{h}^{2}}\left(1-\frac{M^{2}}{m_{A}^{2}}\right)^{3},
$$

where $\sin (\beta-\alpha)=1$ is taken. The deviation from the SM results can be very large when $M^{2} \simeq 0$. The full calculation of the vertex function is shown in Ref. [7] ${ }^{5}$. In FIG. 8, the deviation in the effective $h h h$ coupling constant from the SM value is shown as a function of $\sqrt{\hat{s}}$, the energy of $h^{*} \rightarrow h h$ in the THDM [7]. The mass of the SM-like Higgs boson is taken to be $m_{h}=120 \mathrm{GeV}$ (left) and $m_{h}=160 \mathrm{GeV}$ (right). The masses of extra Higgs bosons are taken to be $m_{\Phi}=200 \mathrm{GeV}$ (dotted line), $m_{\Phi}=300 \mathrm{GeV}$ (dashed line), and $m_{\Phi}=400 \mathrm{GeV}$ (long-dashed line), where $m_{\Phi} \equiv m_{H}=m_{A}=m_{H^{ \pm}}$. These effects can be about $120-70 \%$ for $m_{h}=120-160 \mathrm{GeV}$ with $m_{\Phi} \sim 400 \mathrm{GeV}$. FIG. 8 shows that the deviation in the $h h h$ coupling constant can be approximately described by the analysis with a constant shift by the factor of $(1+\Delta \kappa)$.

In FIG. 9, the invariant mass distribution of the differential cross section for $g g \rightarrow h h$ at the LHC is shown in the THDM ${ }^{6}$. The curves are given in the same manner as in FIG. 8 , and the SM predictions are also denoted by solid curves for comparison. Higgs bosons do not couple to gluons at the tree level, so that the one-loop effect of the extra Higgs bosons only appear in the correction to the $h h h$ coupling constant. For larger extra scalar masses $m_{\Phi}=400 \mathrm{GeV}$, peaks can be found in the near threshold region of a Higgs pair, which come from the enhancement of the $h h h$ coupling constant. There are also peaks around $M_{h h} \sim 400$

5 In the case other than $\sin (\beta-\alpha)=1$, the $h$ hh coupling constant can deviate from the SM value at the tree level because of the mixing between $h$ and $H$. The discussion at the one-loop level with including such a mixing effect is also given in Ref. [7]

6 Cases without $\sin (\beta-\alpha)=1$ were considered in Ref. [54]. The $s$-channel resonance effect of new physics particles were also discussed in Ref. [55]. 

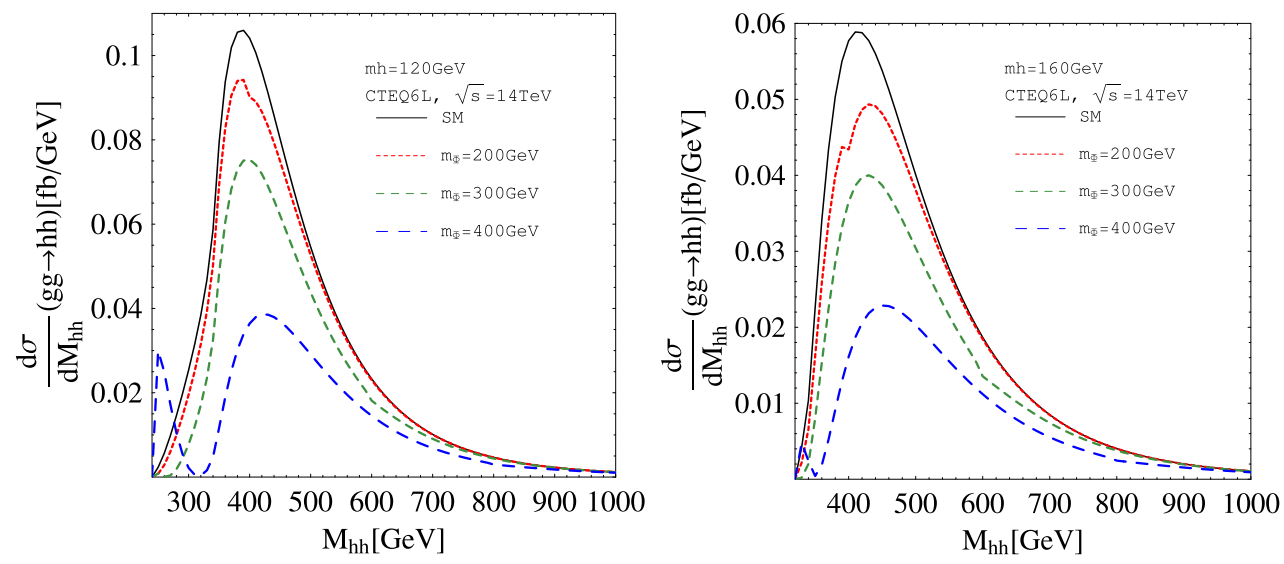

FIG. 9: The invariant mass distribution of $g g \rightarrow h h$ process at the $\mathrm{LHC}$ with $\sqrt{s}=14 \mathrm{TeV}$ for $m_{h}=120 \mathrm{GeV}$ (left) and $m_{h}=160 \mathrm{GeV}$ (right) in the THDM.
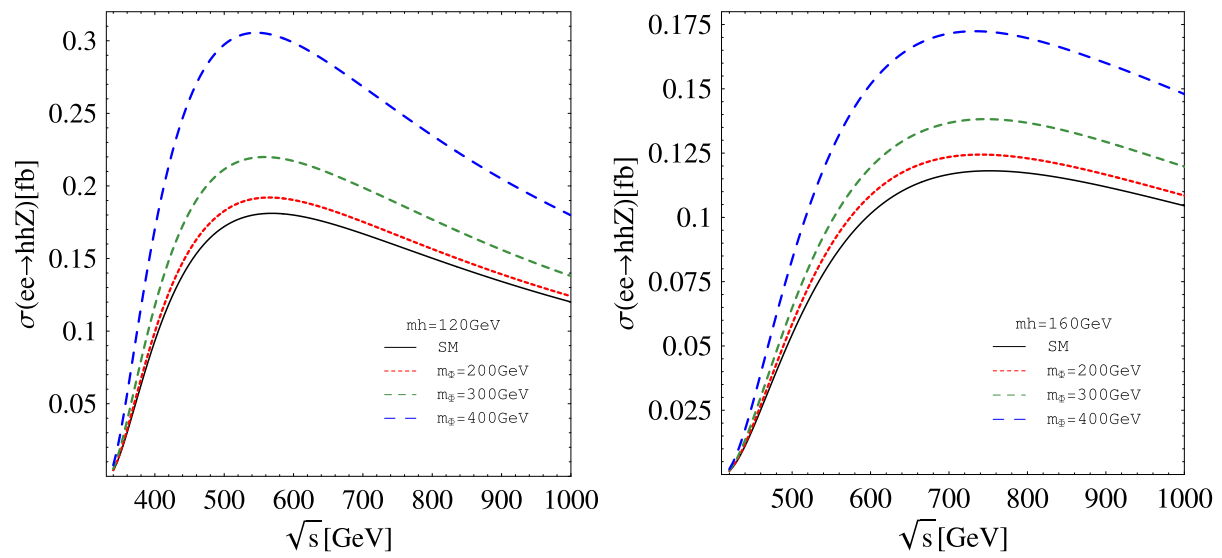

FIG. 10: The cross section of $e^{+} e^{-} \rightarrow h h Z$ process as a function of $\sqrt{s}$ for $m_{h}=120 \mathrm{GeV}$ (left) and $m_{h}=160 \mathrm{GeV}$ (right) in the THDM.

$\mathrm{GeV}$, which are interference effects between the triangular and the box diagrams. Those contributions weaken each other, and hence the enhancement of the $h h h$ coupling constant decreases the cross section as in the SM with constant deviation.

In FIG. 10, we show the cross section of the process $e^{+} e^{-} \rightarrow h h Z$ as a function of the collision energy $\sqrt{s}$ in the THDM ${ }^{7}$. The curves are presented in the same manner as in FIG. 9. Relatively large nondecoupling effect of the extra scalar bosons can appear in the radiative correction to the $h h h$ coupling constant.

In FIG. 11, the cross sections of the Higgs pair production at the PLC are given for the THDM [57 59]. The extra Higgs boson can contribute to the corrections of the $h h h$ coupling constant as well as $\gamma \gamma \rightarrow h h$ process. The $h h h$ coupling constant can be probed by choosing the collision energy to be near threshold region for relatively heavy extra Higgs bosons $m_{\Phi} \gtrsim 400 \mathrm{GeV}$. There are threshold enhancement from the box diagrams after

\footnotetext{
7 More general types of double Higgs-boson production processes at $e^{+} e^{-}$colliders were studied in Ref. [56].
} 

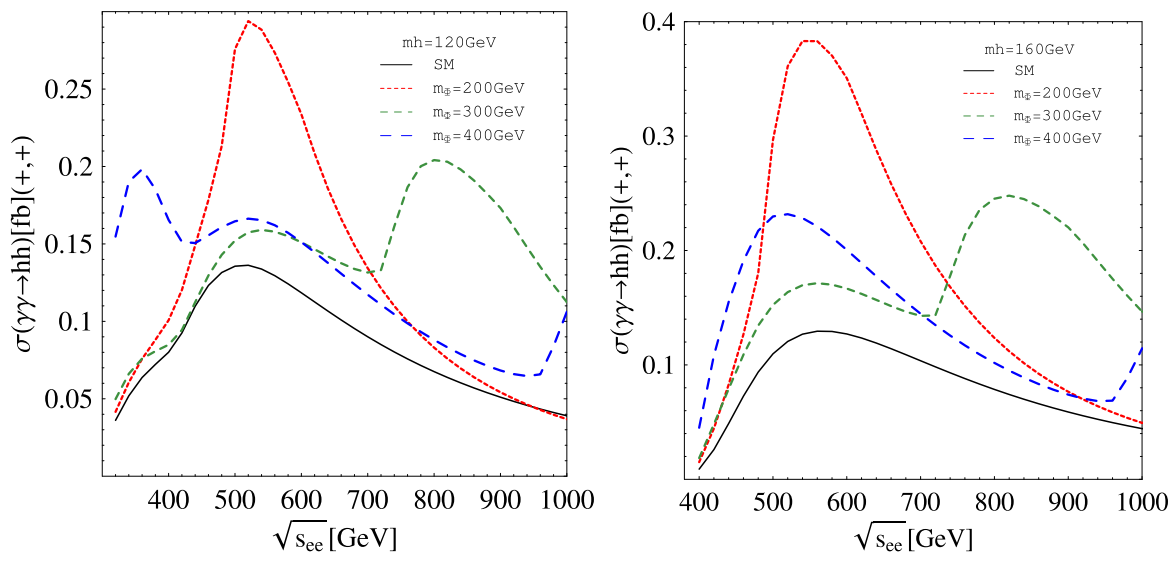

FIG. 11: The cross section of $\gamma(+) \gamma(+) \rightarrow h h$ process at the photon collider option at the ILC as a function of the $e^{-} e^{-}$collision energy for $m_{h}=120 \mathrm{GeV}$ (left) and $m_{h}=160 \mathrm{GeV}$ (right) in the THDM.
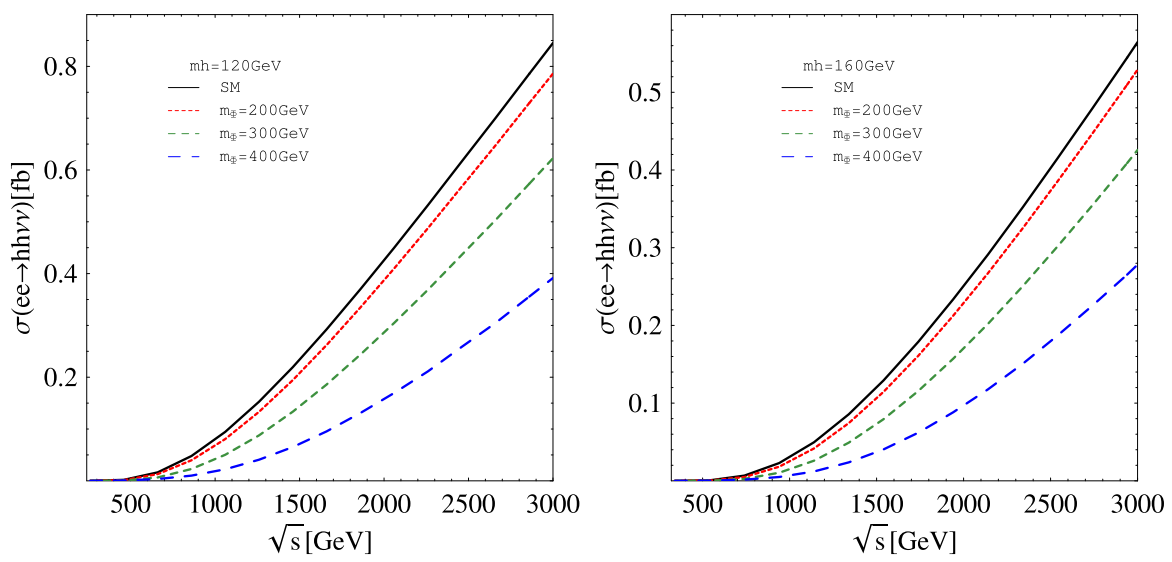

FIG. 12: The cross sections of $e^{+} e^{-} \rightarrow h h \nu \bar{\nu}$ process at the ILC as a function of collision energy $\sqrt{s}$ for $m_{h}=120 \mathrm{GeV}$ (left) and $m_{h}=160 \mathrm{GeV}$ (right) in the THDM.

$\sqrt{s_{e e}} \sim 2 m_{H^{ \pm}}$. The details are shown in Ref. [58].

In FIG. 12, the cross sections for $e^{+} e^{-} \rightarrow h h \nu \bar{\nu}$ with the one-loop corrected $h h h$ coupling constant due to extra scalars are shown. As we show in FIG. 8, the $h$ h $h$ coupling constant can deviate from the SM prediction significantly for $M_{h h} \lesssim 2 m_{\Phi}$, while for $M_{h h} \gtrsim 2 m_{\Phi}$ the deviation becomes small where $M_{h h}$ varies from $2 m_{h}$ to $\sqrt{s}$. We find that the large corrections in low $M_{h h}$ region can enhance the cross section by a factor of a few in magnitude. Although the positive one-loop correction decreases the cross section, this process is still important because the total cross section can be larger than those in other Higgs pair production processes. 


\section{B. Scalar Leptoquarks}

We next consider contributions to cross sections from scalar leptoquarks [60]. Unlike the case with the extra Higgs scalar doublet, the scalar leptoquarks are colored fields. They, therefore, can affect the $g g h$ and the $g g h h$ vertices at the one-loop level, by which the cross section of $g g \rightarrow h h$ in this model can differ from the SM prediction in addition to the effect of the deviation in the $h h h$ vertex.

We here introduce a complex scalar, leptoquark, $\phi_{I_{Q}}=(\overline{\mathbf{3}}, \mathbf{1})_{1 / 3}$ or $(\overline{\mathbf{3}}, \mathbf{1})_{4 / 3}$, as an example for such theories, where $S U(3)_{C}, S U(2)_{L}$ and $U(1)_{Y}$ quantum numbers are shown. The most general scalar potential can be written as

$$
V_{\mathrm{LQ}}\left(\Phi, \phi_{\mathrm{LQ}}\right)=\lambda\left(|\Phi|^{2}-\frac{v^{2}}{2}\right)^{2}+M_{L \mathrm{Q}}^{2}\left|\phi_{\mathrm{IQ}}\right|^{2}+\lambda_{\mathrm{LQ}}\left|\phi_{\mathrm{IQ}}\right|^{4}+\lambda^{\prime}\left|\phi_{\mathrm{IQ}}\right|^{2}|\Phi|^{2},
$$

where $\Phi$ is the SM Higgs doublet. The mass of leptoquarks is given by $m_{\phi_{L Q}}^{2}=M_{L Q}^{2}+\frac{\lambda^{\prime} v^{2}}{2}$.

The searches for the leptoquarks have been performed at the collider experiments at LEP, HERA and Tevatron. In order to avoid large contributions to lepton flavor violating processes, the leptoquarks are usually assumed to be coupled only with one fermion generation in their mass eigenbasis. Under this assumption, the experimental bounds are evaluated as $m_{\phi_{L Q}} \gtrsim 256 \mathrm{GeV}$ [61], $316 \mathrm{GeV}$ [62], and $229 \mathrm{GeV}$ [63] for the leptoquarks interacting only with the first, second, and third generation, respectively. There are indirect limits for masses and their Yukawa couplings through the effective four-fermion interaction 64.

In the large mass limit of an $S U(2)_{L}$ singlet scalar leptoquark, the coupling strength of the effective $g g h$ vertex is enhanced by the factor of $4 / 3$ as compared to the SM prediction, and hence the cross section of the single Higgs boson production $g g \rightarrow h$ can be enhanced approximately by $16 / 9$. The SM Higgs boson with mass $162-166 \mathrm{GeV}$ has been ruled out by analyzing $g g \rightarrow h \rightarrow W W^{(*)}$ process at the Tevatron [3]. The exclusion band of the Higgs boson mass from Tevatron results can be translated a wider range as 155-185 GeV.

The one-loop correction to the hhh coupling constant due to the scalar leptoquark can be calculated analogous to the charged Higgs boson contribution in the THDM [7] as

$$
\frac{\Gamma_{h h h}^{L \mathrm{Q}}}{\Gamma_{h h h}^{\mathrm{SM}}} \simeq 1+\frac{N_{c} m_{\phi_{L Q}}^{4}}{6 \pi^{2} v^{2} m_{h}^{2}}\left(1-\frac{M_{L \mathrm{Q}}^{2}}{m_{\phi_{L Q}}^{2}}\right)^{3} .
$$

The full expression of the one-loop corrected vertex $\Gamma_{h h h}^{L Q}\left(\hat{s}, m_{h}^{2}, m_{h}^{2}\right)$ is given in Appendix A. In FIG. 13, we evaluate the relative size of the one-loop contributions to the $h h h$ coupling constant from the leptoquarks for $m_{h}=120 \mathrm{GeV}$ (left) and for $m_{h}=185 \mathrm{GeV}$ (right). Three reference values are taken for leptoquark masses as $m_{\phi_{L Q}}=256 \mathrm{GeV}$ (dotted line), $m_{\phi_{L Q}}=300 \mathrm{GeV}$ (dashed line), and $m_{\phi_{L Q}}=400 \mathrm{GeV}$ (long-dashed line). For $m_{h}=120$ $\mathrm{GeV}$ the one-loop correction can be about $150 \%$ by a singlet leptoquark with $m_{\phi_{L Q}}=400$ $\mathrm{GeV}$, while for $m_{h}=185 \mathrm{GeV}$ it can be about $60 \%$. These effects are constructive to the SM value in the nondecoupling region $M_{L \mathrm{Q}}^{2} \simeq 0$, which can be significant for heavy leptoquarks because of the $m_{\phi_{L Q}}^{4}$ enhancement. Below the thresholds of $2 m_{\phi_{L Q}}$ the quantum effects are approximately flat for the function of the off-shell Higgs boson energy. 

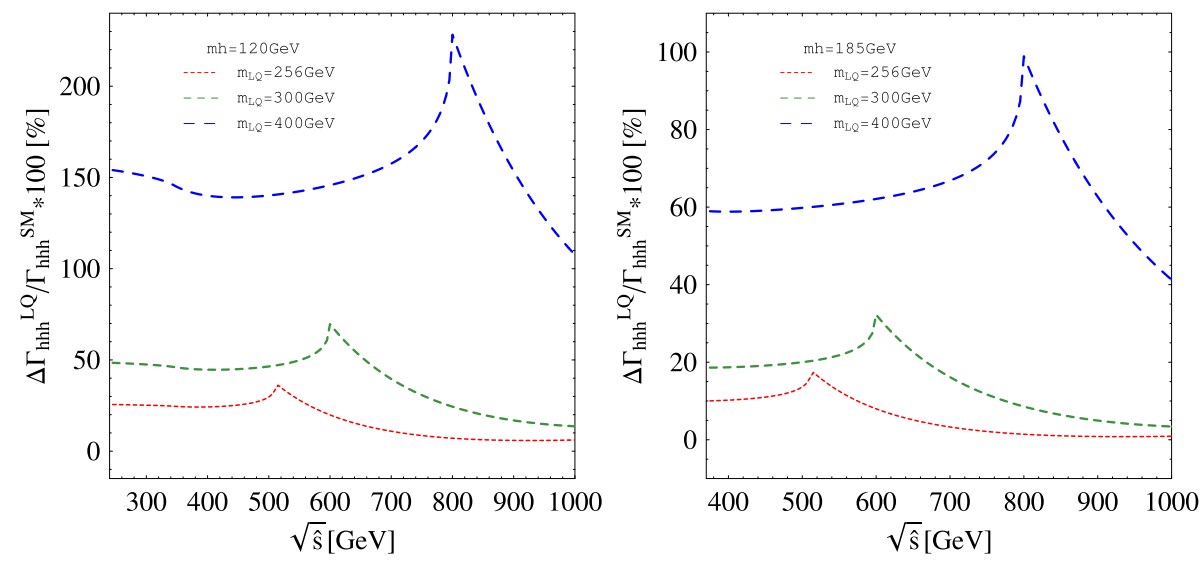

FIG. 13: The rates for one-loop contributions of an $S U(2)$ singlet scalar leptoquark to the $h h h$ coupling constant for $m_{h}=120 \mathrm{GeV}$ (left) and for $m_{h}=185 \mathrm{GeV}$ (right).
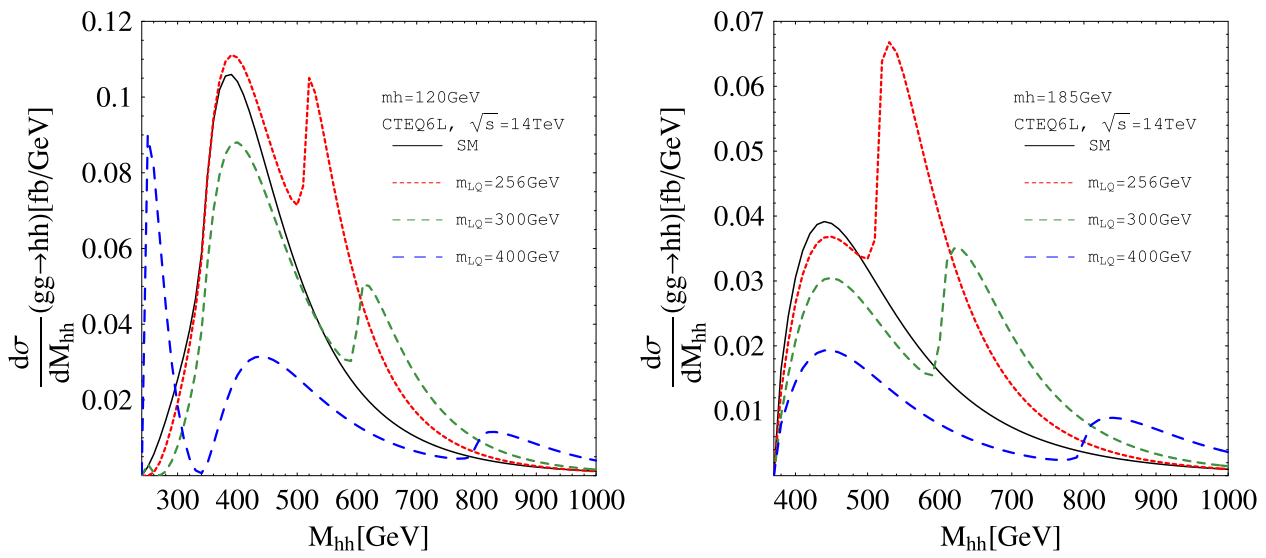

FIG. 14: The invariant mass distribution of $g g \rightarrow h h$ process at the $\mathrm{LHC}$ with $\sqrt{s}=14 \mathrm{TeV}$ for $m_{h}=120 \mathrm{GeV}$ (left) and $m_{h}=185 \mathrm{GeV}$ (right) in the leptoquark model.

In FIG. 14, we show the invariant mass distribution of the cross section for $g g \rightarrow h h$ process at the LHC. For $m_{h}=120 \mathrm{GeV}$ with relatively larger leptoquark masses $m_{\phi} \gtrsim 300$ $\mathrm{GeV}$, peaks can be found in the near threshold region of a Higgs boson pair, which come from the enhancement of the $h h h$ coupling constant as well as that of the $g g h$ coupling constant due to the new colored particles in the triangular diagram: see FIG. 1 for the reference. The threshold enhancement of the on-shell leptoquark pair production can also be seen around $M_{h h} \simeq 2 m_{\phi}$.

In FIG. 15, the cross sections for $e^{+} e^{-} \rightarrow h h Z$ are shown in the case with $m_{h}=120$ $\mathrm{GeV}$ as a function of the collision energy $\sqrt{s}$ for various $m_{\phi_{L Q}}$. The effects of the scalar leptoquarks only appear in the $h$ hh coupling constant.

In FIG. 16, we show the cross section of the Higgs pair production process at a PLC. The effects of the scalar leptoquarks depend on not only their masses but also their electric charges. The first peaks around the threshold region come from the modifications of the $h h h$ coupling constant and the effective $\gamma \gamma h$ vertex due to the scalar leptoquarks. The 

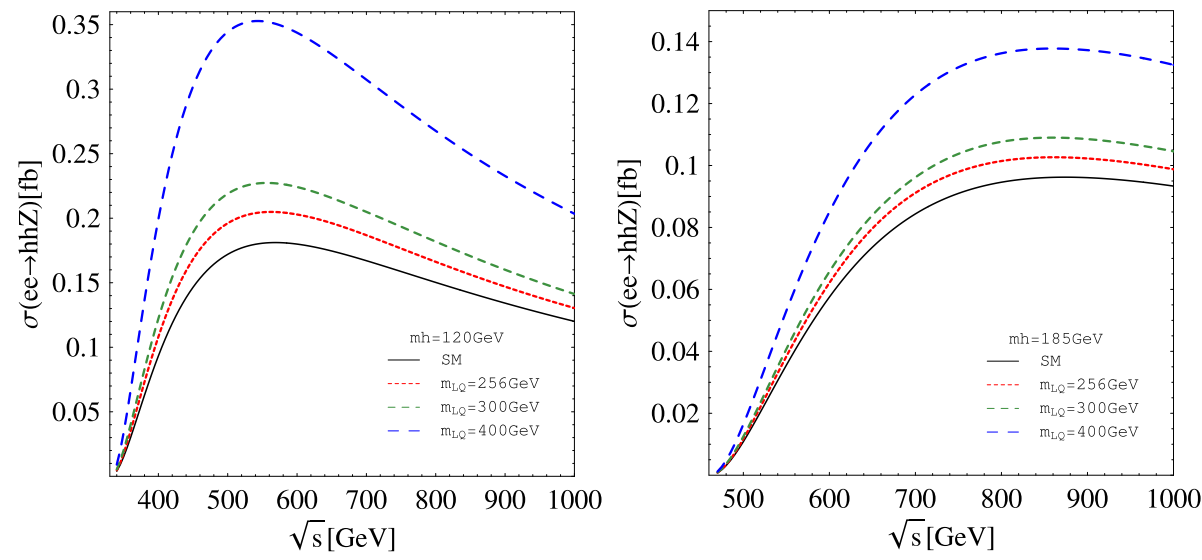

FIG. 15: The cross section of $e^{+} e^{-} \rightarrow h h Z$ process as a function of $\sqrt{s}$ for $m_{h}=120 \mathrm{GeV}$ (left) and $m_{h}=185 \mathrm{GeV}$ (right) in leptoquark models.
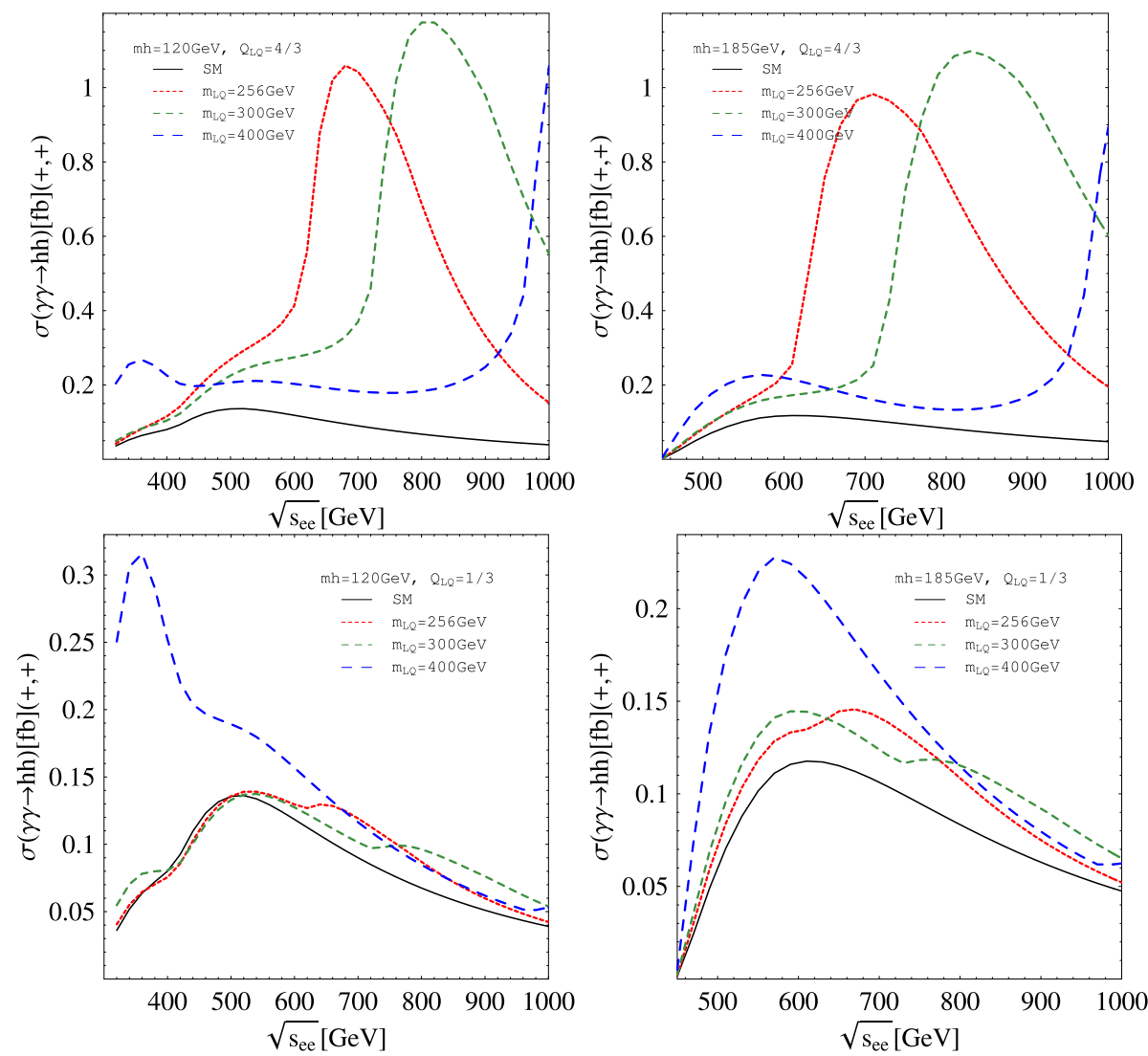

FIG. 16: The cross section of $\gamma(+) \gamma(+) \rightarrow h h$ process at the photon collider option at the ILC as a function of the $e^{-} e^{-}$collision energy for $m_{h}=120 \mathrm{GeV}$ (left column) and $m_{h}=185 \mathrm{GeV}$ (right column). The electric charges for scalar leptoquarks are taken as $Q=4 / 3$ (top) and $Q=1 / 3$ (bottom), respectively. 

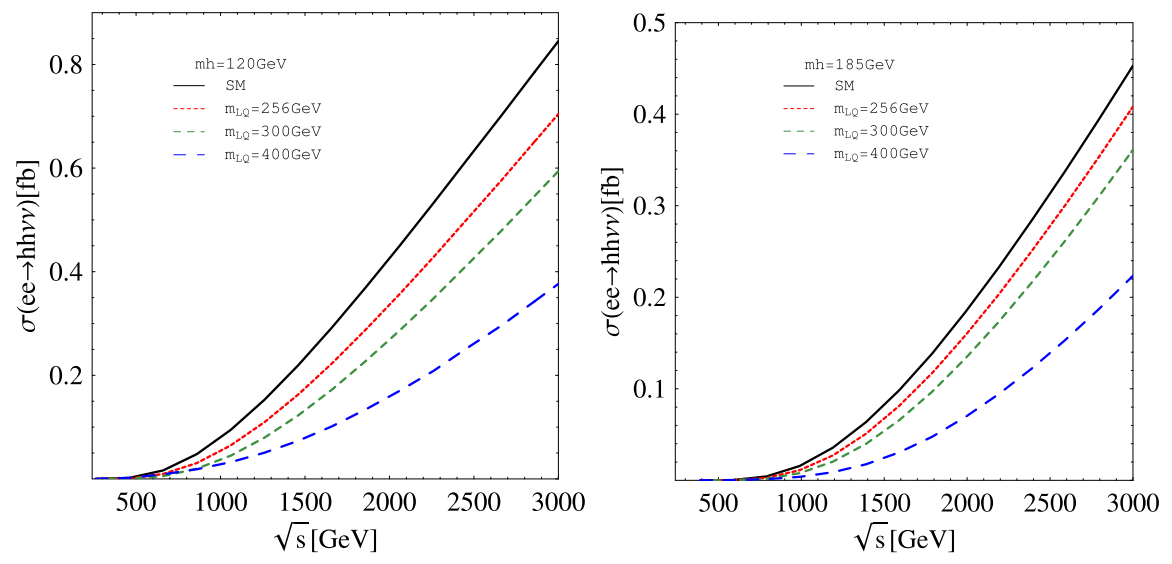

FIG. 17: The cross sections of $e^{+} e^{-} \rightarrow h h \nu \bar{\nu}$ process at the ILC as a function of collision energy $\sqrt{s}$ for $m_{h}=120 \mathrm{GeV}$ (left) and $m_{h}=185 \mathrm{GeV}$ (right) in leptoquark models.

former effect does not depend on the quantum number, while the latter does. The threshold enhancements of the box diagram can be found after $\sqrt{s_{e e}} \sim 2 m_{\phi}$ which are also dependent on the electric charges.

In FIG. 17, the cross section for $e^{+} e^{-} \rightarrow h h \nu \bar{\nu}$ is shown for $m_{h}=120 \mathrm{GeV}$ (left) and $m_{h}=185 \mathrm{GeV}$ (right) with the $h$ hh coupling corrections of leptoquarks. The cross section becomes smaller as in the THDM because of the negative interference.

\section{Chiral fourth generation}

One of the fundamental questions in the SM is the number of generation (family) of quarks and leptons. There is no theoretical reason to restrict the fermion families to be three. The electroweak precision data also do not exclude completely existence of the sequential fourth generation. The fourth generation quarks are colored particles so that they, similarly to the case of scalar leptoquarks, affect the $g g h$ and the $g g h h$ vertices. In addition, the chiral fermion has the nondecoupling property; i.e., the mass is completely proportional to the $\mathrm{VEV}$, so that the one-loop correction to the $h h h$ coupling constant can be very large in magnitude.

We here introduce a sequential set of fermions, i.e., $Q^{\prime}=\left(t^{\prime}{ }_{L}, b_{L}^{\prime}\right)^{T}, L^{\prime}=$ $\left(\ell_{L}^{\prime}, \nu_{L}^{\prime}\right)^{T}, t_{R}^{\prime}, b_{R}^{\prime}, \ell_{R}^{\prime}, \nu_{R}^{\prime}$, as the chiral fourth generation model (Ch4). Neutrinos are assumed to be Dirac particle whose masses are generated by Yukawa interaction. From LEP results, a lower bound on the extra charged lepton $\left(\ell^{\prime}\right)$ mass is $100.8 \mathrm{GeV}$, while for heavy neutral lepton $\left(\nu^{\prime}\right)$ with Dirac nature to be $90.3 \mathrm{GeV}$. The fourth generation up-type quark $\left(t^{\prime}\right)$ is rather stringently constrained, $m_{t^{\prime}} \gtrsim 256 \mathrm{GeV}$, by Tevatron. This bound is independent of the Cabibbo-Kobayashi-Maskawa (CKM) elements between the first three SM fermions and the fourth generation. A similar limit for the heavy down-type quark $\left(b^{\prime}\right)$ is obtained as $m_{b^{\prime}} \gtrsim 128(268) \mathrm{GeV}$ by using the charged (neutral) current decay modes [64]. Further stronger bounds can be found in Ref. [65] by assuming additional assumptions. 


\begin{tabular}{|c||c|c|c|}
\hline$(\widehat{S}, \widehat{T})$ & $m_{t^{\prime}}-m_{b^{\prime}}=50 \mathrm{GeV}$ & $m_{t^{\prime}}-m_{b^{\prime}}=55 \mathrm{GeV}$ & $m_{t^{\prime}}-m_{b^{\prime}}=60 \mathrm{GeV}$ \\
\hline \hline$m_{t^{\prime}}=256 \mathrm{GeV}$ & $(0.18,0.18)$ & $(0.18,0.22)$ & $(0.17,0.26)$ \\
\hline$m_{t^{\prime}}=300 \mathrm{GeV}$ & $(0.19,0.18)$ & $(0.18,0.22)$ & $(0.18,0.26)$ \\
\hline$m_{t^{\prime}}=400 \mathrm{GeV}$ & $(0.19,0.18)$ & $(0.19,0.22)$ & $(0.19,0.26)$ \\
\hline
\end{tabular}

TABLE I: The contributions from the chiral fourth generation fermions to $\widehat{S}$ and $\widehat{T}$ are shown. The mass degeneracies $m_{t^{\prime}}=m_{\ell^{\prime}}$ and $m_{b^{\prime}}=m_{\nu^{\prime}}$ are assumed.

The contributions from the fourth generation fermions to the oblique electroweak parameters can be significant. In the limit of heavy fermions, the $\widehat{S}$-parameter is calculated as

$$
\widehat{S}=\frac{2}{3 \pi}-\frac{1}{6 \pi}\left(\ln \frac{m_{t^{\prime}}^{2}}{m_{b^{\prime}}^{2}}-\ln \frac{m_{\nu^{\prime}}^{2}}{m_{\ell^{\prime}}^{2}}\right) .
$$

It is noted that a complete set of the four generation fermions with degenerate masses is excluded at $6 \sigma$ level, if we take into account the constraint only from the $\widehat{S}$-parameter [64, 66]. It can be relaxed by requiring the preferable mass hierarchy of the fourth generation fermions i.e., $m_{t^{\prime}} \gtrsim m_{b^{\prime}}$ and $m_{\ell^{\prime}} \gtrsim m_{\nu^{\prime}}$ [14]. Furthermore, the breaking of isospin symmetry in the fourth generation doublet gives substantial positive contribution to the $\widehat{T}$-parameter, which pulls back the model to the allowed region in $(\widehat{S}, \widehat{T})$ plane. For $m_{h}=117 \mathrm{GeV}$, the $95 \%$ CL upper bounds are given as $\widehat{S} \leq 0.16$ and $\widehat{T} \leq 0.21$ with a strong correlation [64, 66]. In Table I, the contributions from the fourth generation fermions to oblique parameters are listed. In order to reduce the number of parameters, $m_{t^{\prime}}=m_{\ell^{\prime}}$ and $m_{b^{\prime}}=m_{\nu^{\prime}}$ are taken. We can see that the appropriate mass difference $\left(m_{t^{\prime}}-m_{b^{\prime}}=50-55 \mathrm{GeV}\right)$ between the fourth generation fermions fits the constraint from the electroweak precision data.

From a theoretical point of view, there are a few constraints such as triviality bounds and vacuum stability bounds on the coupling constants [67]. To avoid the Landau pole for the fourth generation Yukawa coupling constant, the mass should be lighter than about 577 $\mathrm{GeV}$ when the cutoff scale of theory is taken to be $2 \mathrm{TeV}$. Instability of the vacuum gives more serious bound on the Higgs boson mass. It may be evaded by introduction of the extra Higgs doublet [67] or some other new physics dynamics, and hence we here keep the mass of the (SM-like) Higgs boson to be the electroweak scale.

The additional heavy colored particles enhance the effective $g g h$ coupling approximately by a factor of 3 which leads to enhancement of the cross section of $g g \rightarrow h$ by a factor of 9 at hadron colliders. Tevatron bounds on the SM Higgs boson mass [3] can be translated into a wider exclusion band as 125-200 GeV for the Higgs boson mass in the chiral fourth generation model [68].

The large one-loop effect on the hhh coupling constant in the SM is generalized straightforwardly to the chiral fourth generation model:

$$
\frac{\Gamma_{h h h}^{\mathrm{Ch} 4}}{\Gamma_{h h h}^{\mathrm{SM}}} \simeq 1-\sum_{f^{\prime}=t^{\prime}, b^{\prime}, \ell^{\prime}, \nu^{\prime}} \frac{N_{c} m_{f^{\prime}}^{4}}{3 \pi^{2} v^{2} m_{h}^{2}} .
$$



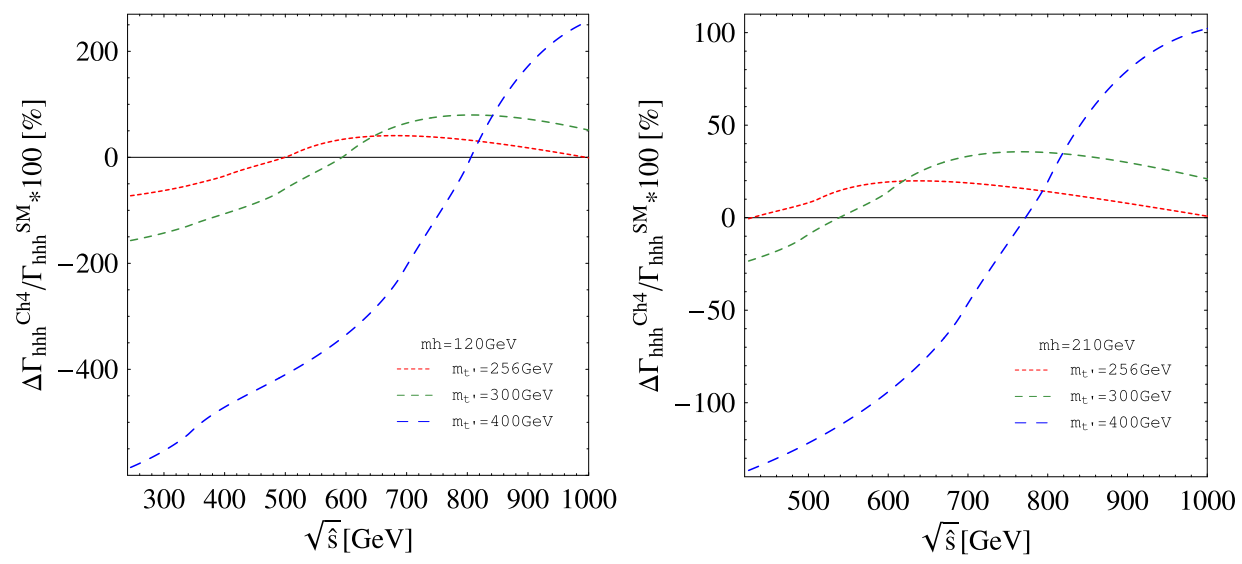

FIG. 18: The rates for one-loop contributions of the chiral fourth generation fermions to the $h h h$ coupling constant for $m_{h}=120 \mathrm{GeV}$ (left) and for $m_{h}=210 \mathrm{GeV}$ (right). The dotted, dashed, long-dashed curved lines indicate masses of heavy fermions as $m_{t^{\prime}}=256,300,400 \mathrm{GeV}$, respectively, with the appropriate mass difference $m_{t^{\prime}}-m_{b^{\prime}}=55 \mathrm{GeV}$.

The explicit formula of $\Gamma_{h h h}^{\mathrm{Ch} 4}$ including energy dependence is given in Appendix A. Since $m_{f^{\prime}}^{4}$ enhancements come from extra heavy fermions, we would expect large quantum corrections to the $h h h$ coupling constant. We note that these fermion loop contributions are always negative to the SM prediction. In FIG. 18, effects of the chiral fourth generation fermions on the $h h h$ coupling constant are shown for $m_{h}=120 \mathrm{GeV}$ (left) and for $m_{h}=210 \mathrm{GeV}$ (right). Hereafter, the mass differences are fixed to be $m_{t^{\prime}}-m_{b^{\prime}}=55 \mathrm{GeV}$ with $m_{t^{\prime}}=m_{\ell^{\prime}}$ and $m_{b^{\prime}}=m_{\nu^{\prime}}$. The masses of the fourth generation up-type quark are taken as three representative values, $m_{t^{\prime}}=256 \mathrm{GeV}$ (dotted line), $300 \mathrm{GeV}$ (dashed line), and $400 \mathrm{GeV}$ (long-dashed line). The hhh coupling constant is changed significantly depending on the energy of the off-shell Higgs boson. In the low energy limit, a huge quantum correction to the hhh coupling constant can be more than 100\%, which can easily overwhelm the SM contribution and change the sign of the total amplitude. We again note that from the vacuum stability condition it is highly disfavored to introduce too heavy fourth generation fermion unless the Higgs sector is extended.

In FIG. 19, we show the invariant mass distribution of the differential cross section of $g g \rightarrow h h$ in the SM with a complete set of fourth generation for $m_{h}=120 \mathrm{GeV}$ (left) and for $m_{h}=210 \mathrm{GeV}$ (right). The production cross sections of the chiral fourth generation model can be 10-100 times larger than that of the SM. The first peaks come not only from the large one-loop correction to the $h h h$ vertex but also from the enhancement of the $g g h$ vertex due to the fourth generation quarks. The threshold enhancements of on-shell fermion-pair production can also be seen around $M_{h h} \simeq 2 m_{t}$ and $M_{h h} \simeq 2 m_{f^{\prime}}$ which are smeared by the distribution of high energy gluons.

In FIG. 20, we show the cross section of the double-Higgs-strahlung process $e^{+} e^{-} \rightarrow h h Z$ as a function of the collision energy. The cross section can be reduced by the suppression of the $h h h$ coupling constant due to the fourth generation fermions. Unlike the case of 

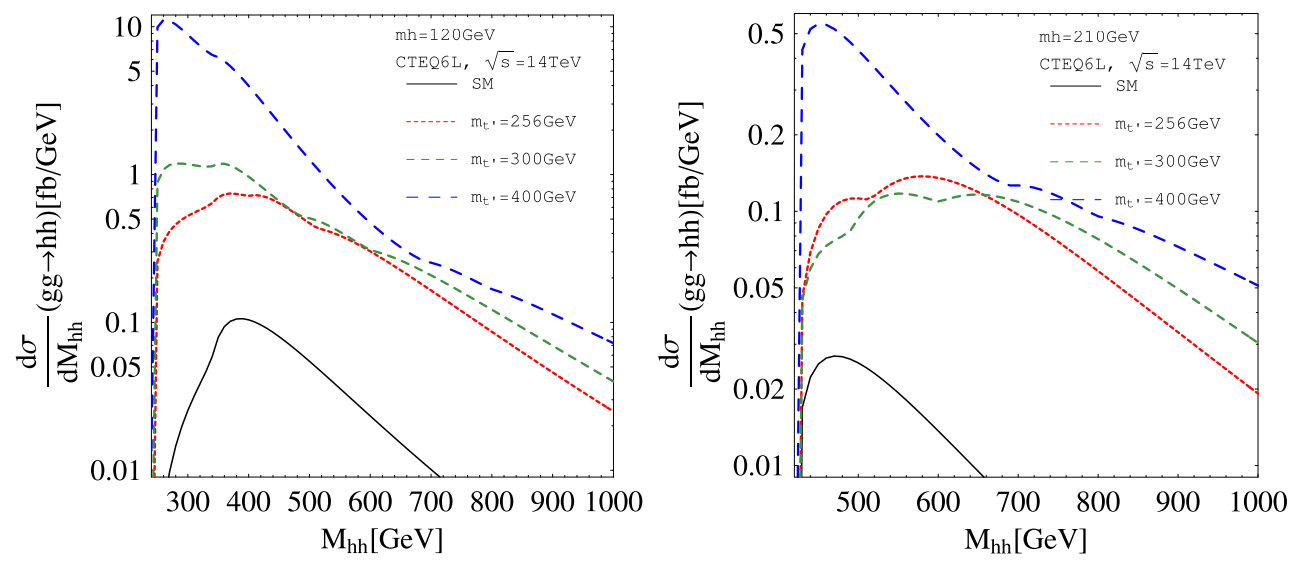

FIG. 19: The invariant mass distribution of $g g \rightarrow h h$ process at the $\mathrm{LHC}$ with $\sqrt{s}=14 \mathrm{TeV}$ for $m_{h}=120 \mathrm{GeV}$ (left) and $m_{h}=210 \mathrm{GeV}$ (right) in the chiral fourth generation model. Three representative values of $t^{\prime}$ mass are chosen as $256 \mathrm{GeV}$ (dotted line), $300 \mathrm{GeV}$ (dashed line), and $400 \mathrm{GeV}$ (long-dashed line). The SM prediction is also shown by a solid curved line for comparison.
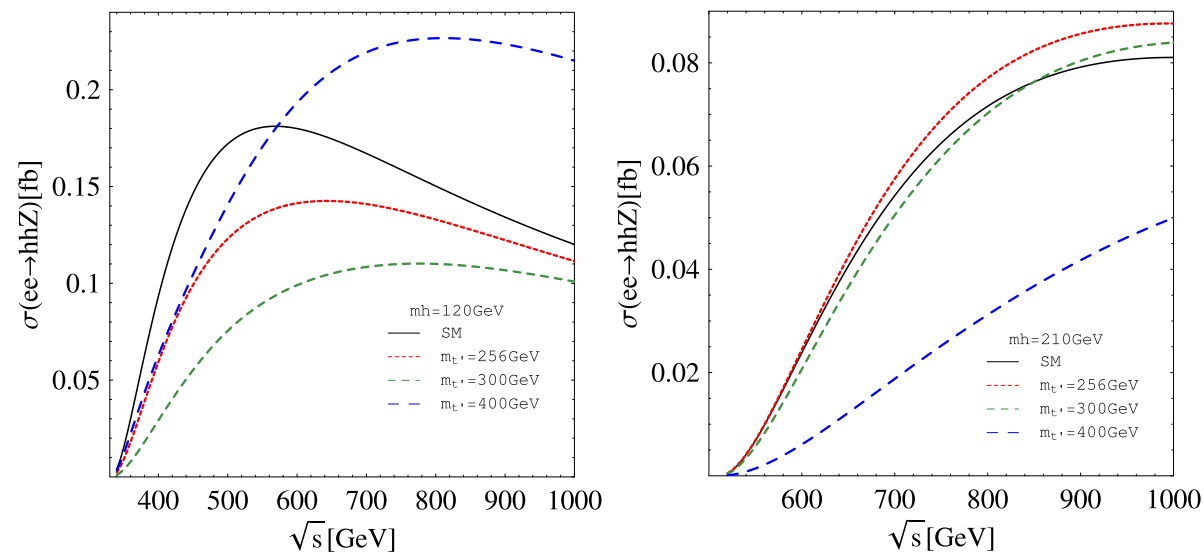

FIG. 20: The cross section of $e^{+} e^{-} \rightarrow h h Z$ process as a function of $\sqrt{s}$ for $m_{h}=120 \mathrm{GeV}$ (left) and $m_{h}=210 \mathrm{GeV}$ (right) in the chiral fourth generation model.

$\Delta \kappa$ approximation the deviation of the cross section from the SM depends on the collision energy, because the quantum corrections to the hhh coupling constant are the function of the energy for the off-shell Higgs boson.

In FIG. 21, the cross section of the Higgs pair production at a photon collider is given as a function of $\sqrt{s_{e e}}$ with $m_{h}=120 \mathrm{GeV}$ (left) and $m_{h}=210 \mathrm{GeV}$ (right). All the fourth generation fermions contribute to the both triangular and the box diagrams (see FIG. 5) due to the large Yukawa coupling constant, which can enhance the cross section significantly by a factor of 10 for the wide range of $\sqrt{s_{e e}}$. The threshold effects of the on-shell heavy fermions can be found soon above the thresholds $\sqrt{s_{e e}} \sim 2 m_{t}$ and $\sqrt{s_{e e}} \sim 2 m_{f^{\prime}}$ because the photon luminosity has a peak around $0.8 \sqrt{s_{e e}}$. At the PLC, we can have larger cross sections even for relatively heavy Higgs bosons.

In FIG. 22, we show the cross section for $e^{+} e^{-} \rightarrow h h \nu \bar{\nu}$ with the one-loop corrected 

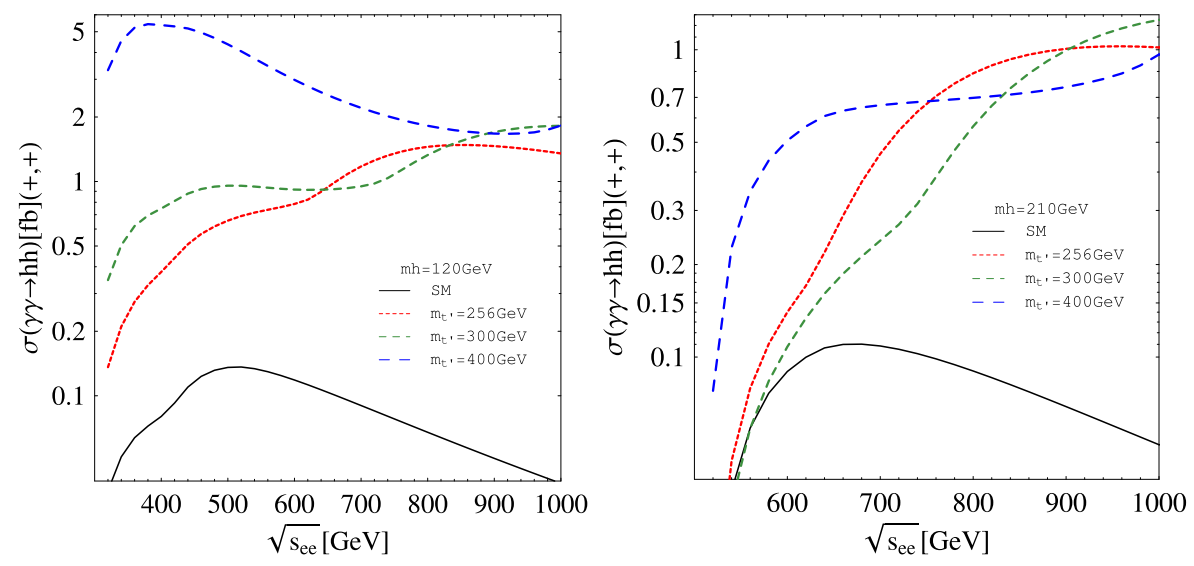

FIG. 21: The cross section of $\gamma(+) \gamma(+) \rightarrow h h$ process at the photon collider option at the ILC as a function of the $e^{-} e^{-}$collision energy for $m_{h}=120 \mathrm{GeV}$ (left) and $m_{h}=210 \mathrm{GeV}$ (right) in the chiral fourth generation model.
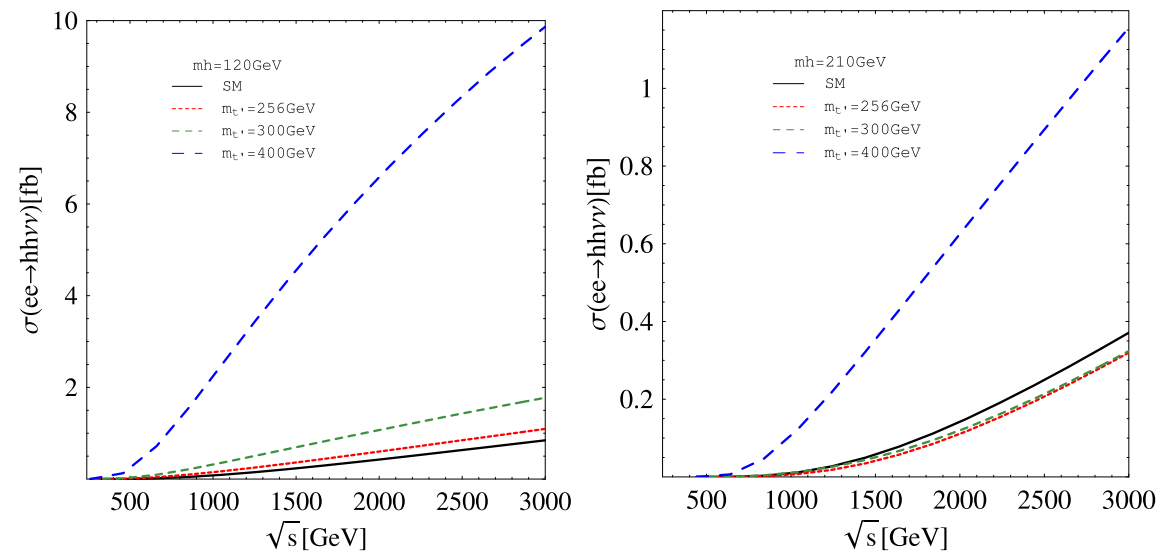

FIG. 22: The cross sections of $e^{+} e^{-} \rightarrow h h \nu \bar{\nu}$ process at the ILC as a function of collision energy $\sqrt{s}$ for $m_{h}=120 \mathrm{GeV}$ (left) and $m_{h}=210 \mathrm{GeV}$ (right) in the chiral fourth generation model.

hhh coupling constant. For $m_{h}=120 \mathrm{GeV}$ (left), the production rate becomes significantly large compared to the SM rate. This enhancement mainly comes from the large quantum corrections to the $h h h$ coupling constant in the smaller $M_{h h}$ region. For $m_{h}=210 \mathrm{GeV}$, corrections to the $h h h$ coupling constant are relatively small. However, for larger $M_{h h}$ values the one-loop correction $\left(\Delta \Gamma_{h h h}^{\mathrm{Ch} 4} / \Gamma_{h h h}^{\mathrm{SM}}\right)$ goes back to negative, and its effect rapidly becomes important for $M_{h h} \gtrsim 1500 \mathrm{GeV}$, which makes the cross section larger.

\section{Vectorlike quarks}

Various types of vectorlike fermions have also been discussed in the literature. They can appear in extra-dimension models with bulk fermions [69], in little Higgs models [70] and in the top seesaw model [71]. As a representative case of these models, we adopt a pair of vectorlike up-type quarks, $T_{0 L}$ and $T_{0 R}$, which transform as $(\mathbf{3}, \mathbf{1})_{2 / 3}$ under the gauge 
symmetry.

The Lagrangian relevant to the mass of the SM top-quark and the vectorlike up-type quark can be written as

$$
\mathcal{L}^{\text {mass }}=-y_{t} \bar{Q}_{0} t_{0 R} \widetilde{\Phi}-Y_{T} \bar{Q}_{0} T_{0 R} \widetilde{\Phi}-M_{T} \bar{T}_{0 L} T_{0 R}+\text { H.c. }
$$

where we have dropped the terms proportional to $\bar{T}_{0 L} t_{0 R}$ which are absorbed by redefinitions of $t_{0 R}$ and $T_{0 R}$ without loss of generality. Since the $t_{0}-T_{0}$ mixing term is allowed by the symmetry, $t_{0}$ and $T_{0}$ are no longer mass eigenstates. The mass eigenstates $t$ and $T$ are determined by diagonalization of the mass matrix,

$$
\widehat{M}_{t}=\left(\begin{array}{cc}
\frac{y_{t} v}{\sqrt{2}} & \frac{Y_{T} v}{\sqrt{2}} \\
0 & M_{T}
\end{array}\right)=U_{L}^{\dagger}\left(\begin{array}{ll}
m_{t} & \\
& m_{T}
\end{array}\right) U_{R}
$$

where

$$
\left(\begin{array}{c}
t_{X} \\
T_{X}
\end{array}\right)=U_{X}\left(\begin{array}{c}
t_{0 X} \\
T_{0 X}
\end{array}\right)=\left(\begin{array}{cc}
c_{X} & -s_{X} \\
s_{X} & c_{X}
\end{array}\right)\left(\begin{array}{c}
t_{0 X} \\
T_{0 X}
\end{array}\right), \quad \text { where } X=L, R
$$

The direct search for the vectorlike quarks has been performed [64]. Their main production modes at hadron colliders are $g g \rightarrow \bar{T} T$, so that the lower bound of the up-type vectorlike fermion is basically the same as the fourth generation up-type quark, $m_{T} \gtrsim 256$ $\mathrm{GeV}$.

The vectorlike quarks are also severely constrained by electroweak precision data [72]. For $\widehat{U}=0$, the experimental values for oblique parameters are $\widehat{S}=-0.04 \pm 0.09$ and $\widehat{T}=0.02 \pm 0.09$ where $m_{h}=117 \mathrm{GeV}$ is assumed. The contributions to the $\widehat{S}$ parameter due to the vectorlike top-quark $(T)$ are less than 0.004 for $M_{T} \gtrsim 1000 \mathrm{GeV}$, which can be neglected. The $t_{0}-T_{0}$ Yukawa coupling constant is set to be $Y_{T}=1$ throughout this analysis. On the other hand, the $\widehat{T}$ parameter is rather sensitive to the model parameters. The one (two) sigma bound on the lowest value of $M_{T}$ is 1100 (1700) $\mathrm{GeV}$ for $m_{h}=120$ $\mathrm{GeV}$. For $m_{h}=160 \mathrm{GeV}$, these constraints are slightly milder, $M_{T} \gtrsim 1100(1500) \mathrm{GeV}$ at $1 \sigma(2 \sigma)$ confidence level.

The one-loop correction to the $h h h$ coupling constant due to the vectorlike top-quark is evaluated as

$$
\begin{aligned}
\frac{\Gamma_{h h h}^{\mathrm{Vec}}}{\Gamma_{h h h}^{\mathrm{SM}}} \simeq 1 & -\frac{N_{c} m_{T}^{4}}{3 \pi^{2} v^{2} m_{h}^{2}\left(1-m_{t}^{2} / m_{T}^{2}\right)}\left(1-\frac{y_{t}^{\mathrm{eff}}}{\sqrt{2}} \frac{v}{m_{t}}\right)\left\{-\frac{3 m_{t}^{4}}{m_{T}^{4}}\left[3-\frac{m_{t}^{2}}{m_{T}^{2}}-\frac{2}{1-m_{t}^{2} / m_{T}^{2}} \ln \frac{m_{T}^{2}}{m_{t}^{2}}\right]\right. \\
+ & \frac{3 m_{t}^{2}}{m_{T}^{2}}\left[2+5 \frac{m_{t}^{2}}{m_{T}^{2}}-\frac{m_{t}^{4}}{m_{T}^{4}}-\frac{6 m_{t}^{2} / m_{T}^{2}}{1-m_{t}^{2} / m_{T}^{2}} \ln \frac{m_{T}^{2}}{m_{t}^{2}}\right]\left(1-\frac{y_{t}^{\mathrm{eff}}}{\sqrt{2}} \frac{v}{m_{t}}\right) \\
+ & {\left.\left[\left(1+\frac{m_{t}^{2}}{m_{T}^{2}}\right)\left(1-8 \frac{m_{t}^{2}}{m_{T}^{2}}+\frac{m_{t}^{4}}{m_{T}^{4}}\right)+\frac{12 m_{t}^{4} / m_{T}^{4}}{1-m_{t}^{2} / m_{T}^{2}} \ln \frac{m_{T}^{2}}{m_{t}^{2}}\right]\left(1-\frac{y_{t}^{\mathrm{eff}}}{\sqrt{2}} \frac{v}{m_{t}}\right)^{2}\right\}, \quad(20) }
\end{aligned}
$$

where $y_{t}^{\text {eff }}=c_{L}\left(c_{R} y_{t}-s_{R} Y_{T}\right)$. Although there is a $m_{T}^{4}$ enhancement factor, the correction to the $h h h$ coupling constant can not be large. This is because a factor $\left(1-y_{t}^{\text {eff }} v /\left(\sqrt{2} m_{t}\right)\right)$ 

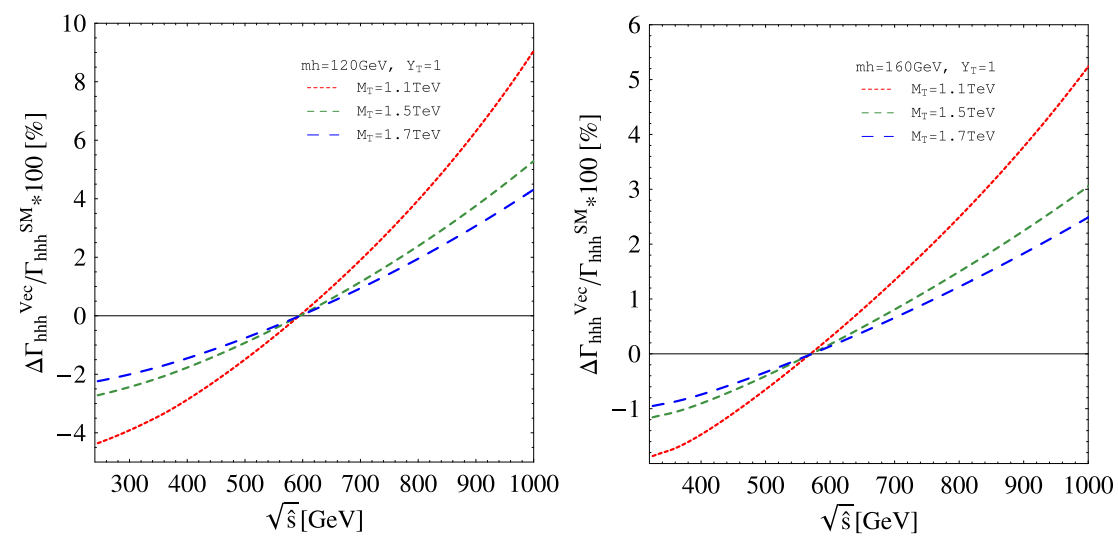

FIG. 23: The rates for one-loop contributions from the vectorlike top-quark $T$ to the $h h h$ coupling constant for $m_{h}=120 \mathrm{GeV}$ (left) and for $m_{h}=160 \mathrm{GeV}$ (right). The $t_{0}-T_{0}$ Yukawa coupling is taken to be $Y_{T}=1$, and the gauge invariant mass parameter $M_{T}$ is chosen as $1100 \mathrm{GeV}$ (dotted line), $1500 \mathrm{GeV}$ (dashed line) and $1700 \mathrm{GeV}$ (long-dashed line), respectively.
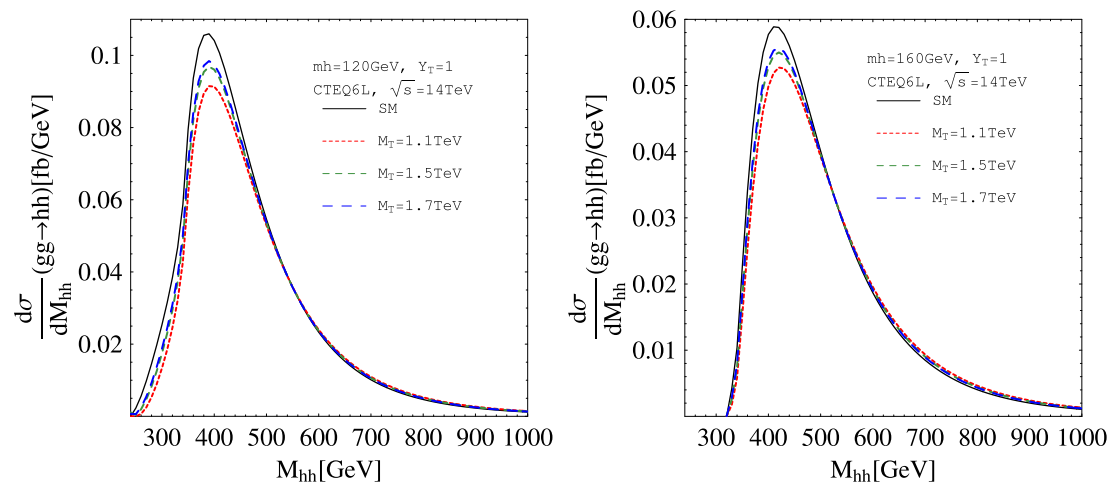

FIG. 24: The invariant mass distribution of $g g \rightarrow h h$ process at the $\mathrm{LHC}$ with $\sqrt{s}=14 \mathrm{TeV}$ for $m_{h}=120 \mathrm{GeV}$ (left) and $m_{h}=160 \mathrm{GeV}$ (right) in the SM with the vectorlike top-quark.

is approximately expressed as $\left(Y_{T} v /\left(\sqrt{2} m_{T}\right)\right)^{2}$ for large $m_{T}$, so that the correction to the $h h h$ coupling constant decouples as $1 / m_{T}^{2}$. In FIG. 23, the effects on the $h h h$ coupling constant due to the vectorlike top-quark are shown. The stringent experimental bounds from the electroweak precision data impose that mass of $T$ particle is heavy, $m_{T} \gtrsim 1100 \mathrm{GeV}$. Therefore, there can be no significant nondecoupling effect on the hhh coupling constant.

In FIG. 24, we show the invariant mass distribution for the cross section of $g g \rightarrow h h$ at the LHC with $\sqrt{s}=14 \mathrm{TeV}$ in the model with the vectorlike top-quark. The vectorlike top-quarks give new contribution to the both triangular and box diagrams. However, the deviations of the cross section from the SM value are at most 5\%, which can not be large because of their decoupling nature. In FIG. 25, the cross sections for the double-Higgsstrahlung process are shown in the model with the vectorlike top-quark. The effects of the vectorlike fermions only appear in the $h h h$ coupling constant. The impact of the vectorlike top-quarks is quite small also in this process. In FIG. 26, we show the cross section of 

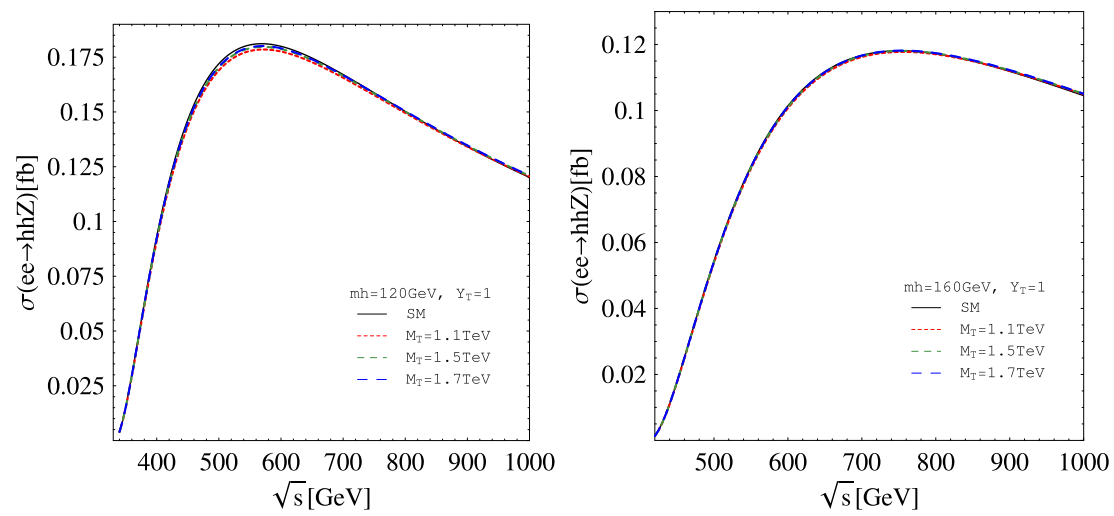

FIG. 25: The cross section of $e^{+} e^{-} \rightarrow h h Z$ process as a function of $\sqrt{s}$ for $m_{h}=120 \mathrm{GeV}$ (left) and $m_{h}=160 \mathrm{GeV}$ (right) in the SM with the vectorlike top-quark.
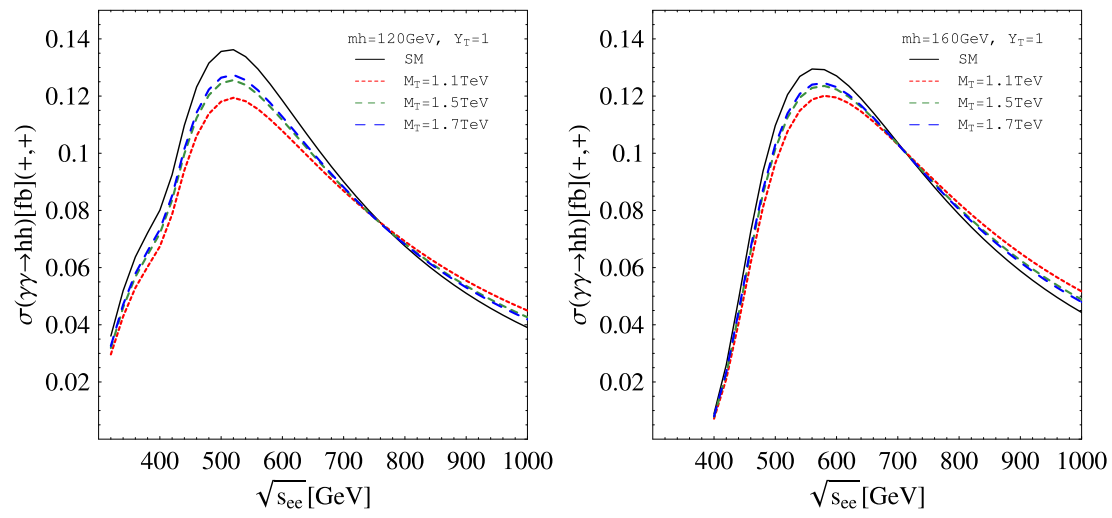

FIG. 26: The cross section of $\gamma(+) \gamma(+) \rightarrow h h$ process at the photon collider option at the ILC as a function of the $e^{-} e^{-}$collision energy for $m_{h}=120 \mathrm{GeV}$ (left) and $m_{h}=160 \mathrm{GeV}$ (right) in the SM with the vectorlike top-quark.

$\gamma \gamma \rightarrow h h$ process in the model with vectorlike top-quark. Similarly to the gluon fusion process $g g \rightarrow h h$, the new physics effects are rather small. In FIG. 27, the cross section for $e^{+} e^{-} \rightarrow h h \nu \bar{\nu}$ is shown as a function of $e^{+} e^{-}$energy. Since the effect on the $h h h$ coupling constant are small in this model, the deviation of cross section is very tiny. We note that the deviations of cross sections for $g g \rightarrow h h$ and $\gamma \gamma \rightarrow h h$ are larger than those for $e^{+} e^{-} \rightarrow h h Z$ and $e^{+} e^{-} \rightarrow h h \nu \bar{\nu}$ due to the flavor changing Yukawa interaction between $t$ and $T$ from the one-loop box diagrams.

\section{SUMMARY AND DISCUSSIONS}

We have studied the double Higgs boson production processes $g g \rightarrow h h, e^{+} e^{-} \rightarrow h h Z$, $\gamma \gamma \rightarrow h h$ and $e^{+} e^{-} \rightarrow h h \nu \bar{\nu}$ in various new physics models. These processes include diagrams that contain the $h h h$ coupling constant, so that they can be used to obtain information of the Higgs potential. SM cross sections for these processes are shown at the leading order in 

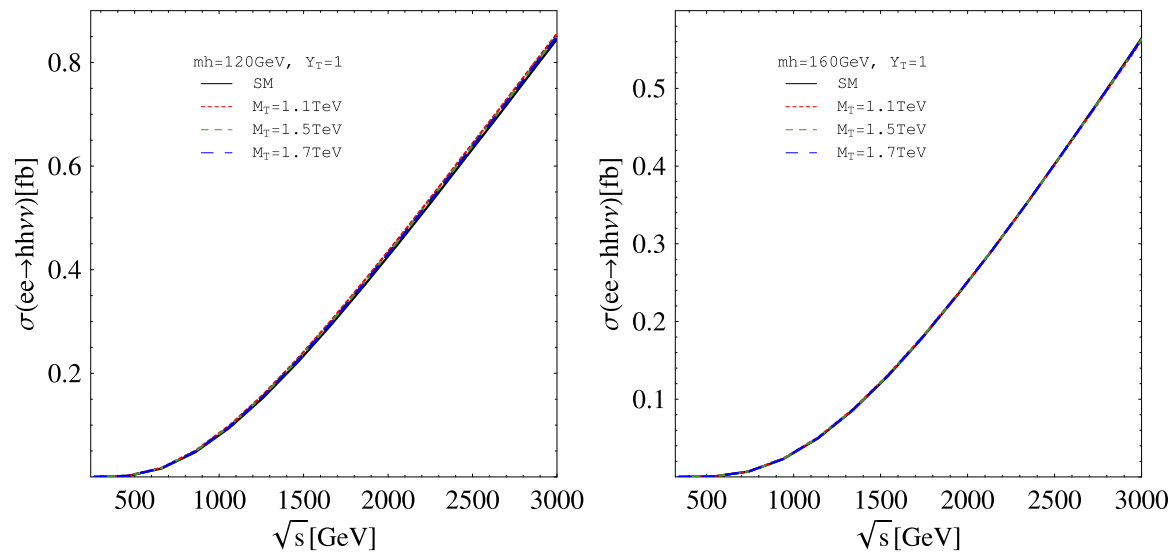

FIG. 27: The cross sections of $e^{+} e^{-} \rightarrow h h \nu \bar{\nu}$ process at the ILC as a function of collision energy $\sqrt{s}$ for $m_{h}=120 \mathrm{GeV}$ (left) and $m_{h}=160 \mathrm{GeV}$ (right) in the SM with vectorlike top-quark.

\begin{tabular}{|c||c|c|c|c|}
\hline$m_{h}[\mathrm{GeV}]$ & $\sigma_{\mathrm{SM}}^{g g \rightarrow h h}[\mathrm{fb}]$ & $\sigma_{\mathrm{SM}}^{e^{+} e^{-} \rightarrow h h Z}[\mathrm{fb}]$ & $\sigma_{\mathrm{SM}}^{\gamma \gamma \rightarrow h h}[\mathrm{fb}]$ & $\sigma_{\mathrm{SM}}^{e^{+} e^{-} \rightarrow h h \nu \bar{\nu}}[\mathrm{fb}]$ \\
\hline \hline 120 & 21 & $0.09-0.2$ & 0.14 & $0.09-0.9$ \\
\hline 160 & 12 & $0-0.05$ & 0.11 & $0.02-0.6$ \\
\hline 185 & 8.0 & $0-0.01$ & 0.07 & $0.02-0.5$ \\
\hline 210 & 5.2 & 0 & $\approx 0$ & $0.01-0.4$ \\
\hline
\end{tabular}

TABLE II: The total cross sections of Higgs boson pair production for $m_{h}=120,160,185$ and 210 $\mathrm{GeV}$ in the $\mathrm{SM}$ are listed. The proton-proton collision energy is taken as $\sqrt{s}=14 \mathrm{TeV}$ for the gluon fusion mechanism. For the double-Higgs-strahlung $\sqrt{s_{e e}}$ is varied from $400 \mathrm{GeV}$ to $500 \mathrm{GeV}$, while for the $W$ boson fusion $\sqrt{s_{e e}}$ is varied from $1 \mathrm{TeV}$ to $3 \mathrm{TeV}$. For photon-photon collisions energy is optimized to obtain the largest cross sections in a range $\sqrt{s_{e e}} \gtrsim 500 \mathrm{GeV}$.

Table@for several values of $m_{h}$. In order to see the impact of a deviation in the $h h h$ coupling constant on these double Higgs boson production processes, we have at first evaluated their cross sections in the SM but assuming the constant deviation in the hhh coupling constant by the factor of $(1+\Delta \kappa)$. The results are summarized as follows:

- The $g g \rightarrow h h$ process is the one-loop induced process, where contributions from the triangle top-loop diagrams with the $h h h$ coupling constant and the box-type toploop diagrams are destructive. Therefore, a negative (positive) deviation in the $h h h$ coupling constant can make the cross section larger (smaller). For example, when $\Delta \kappa=$ -1 , namely the case without the $h h h$ coupling, the cross section can be approximately doubled (tripled) as compared to the SM value with $\Delta \kappa=0$ for $m_{h}=120 \mathrm{GeV}$ (160 $\mathrm{GeV})$.

- For the process of $e^{+} e^{-} \rightarrow h h Z$, on the other hand, the contribution of the tree-level diagram with the $h h h$ coupling constant and that of the other tree-level diagrams are constructive, so that the cross section is enhanced by the positive deviation in the $h h h$ coupling constant. Because of an $s$-channel process, as seen in Table II, the cross 
section becomes rapidly smaller for a larger mass of the Higgs boson, so that this process may only be useful for a light Higgs bosons like $m_{h} \lesssim 140 \mathrm{GeV}$ for $\sqrt{s} \simeq 500$ $\mathrm{GeV}$.

- The one-loop induced process $\gamma \gamma \rightarrow h h$ can play a complementary role to $g g \rightarrow h h$ and $e^{+} e^{-} \rightarrow h h Z$. For $\sqrt{s_{e e}} \lesssim 500 \mathrm{GeV}$, the sensitivity to the deviation in the $h h h$ coupling constant is significant for both $m_{h}=120 \mathrm{GeV}$ and $160 \mathrm{GeV}$. Direction of interference of the diagram with the $h h h$ coupling constant and the other diagrams is however opposite; i.e., destructive and constructive for $m_{h}=120 \mathrm{GeV}$ and 160 $\mathrm{GeV}$, respectively. These characteristic behaviors of $\gamma \gamma \rightarrow h h$ can be complementary to $g g \rightarrow h h$ and $e^{+} e^{-} \rightarrow h h Z$ in the measurement of the $h h h$ coupling constant. Furthermore, sensitivity to the deviation in the $h h h$ coupling constant can be better by using this process even when the collision energy of the linear collider is limited to be relatively low $\left(\sqrt{s_{e e}} \lesssim 500 \mathrm{GeV}\right)$.

- The $W$ boson fusion process $e^{+} e^{-} \rightarrow h h \nu \bar{\nu}$ can be useful for measuring the $h h h$ coupling constant at an energy upgrade of the ILC or the CLIC with $\sqrt{s_{e e}}=1-3 \mathrm{TeV}$, because the production cross section is monotonically increasing with the collision energy due to the $t$-channel colinear effect. The diagram with the $h h h$ coupling constant has the opposite sign with the other diagrams.

We have evaluated cross sections for these processes in various new physics models such as the THDM, the model with scalar leptoquarks, the model with chiral fourth generation quarks, and the model with a vectorlike quark. In these models, apart from the deviated hh coupling constant, additional one-loop diagrams can contribute to the cross sections especially in the loop induced processes $g g \rightarrow h h$ and $\gamma \gamma \rightarrow h h$. Only one-loop diagrams of colored particles contribute to the former process, while those of all the charged particles do to the latter one. In Table III, the results for possible deviations in cross sections for these processes are summarized with the deviation in the $h h h$ coupling constant for several typical values of $m_{h}$ and the collision energies in each model. We summarize the results for each model below in order.

In the THDM, one-loop corrections of additional scalar bosons to the hhh coupling constant can give $+100 \%$ deviations in the SM-like limit where we set $\sin (\beta-\alpha)=1$ with $M^{2} \simeq 0$. This positive large quantum correction to the hhh coupling constant is common in the extended Higgs sectors with nondecoupling property where the mass of the scalar bosons comes mainly from the VEV. The effect of the large deviation in the hhh coupling constant can be well described by the analysis with the constant shift of the hhh coupling constant by the factor of $(1+\Delta \kappa)$. A qualitative difference can be seen in the one-loop induced $\gamma \gamma \rightarrow h h$ process, where one-loop diagrams of charged Higgs bosons can change to the cross section.

In the scalar leptoquark models, the correction to the $h h h$ vertex is positive because of additional bosonic loop contributions, as in the THDM. The magnitude can be larger than $+100 \%$ via the nondecoupling effect of scalar leptoquarks in the loop when $M_{L \mathrm{Q}}^{2} \simeq 0$. 


\begin{tabular}{|c||c|c|c|c|c|c|}
\hline Model & $m_{h}[\mathrm{GeV}]$ & $\frac{\Gamma_{h h h}^{\mathrm{NP}}-\Gamma_{h h h}^{\mathrm{SM}}}{\Gamma_{h h h}^{\mathrm{SM}}}$ & $\Delta r_{\mathrm{NP}}^{g g \rightarrow h h}$ & $\Delta r_{\mathrm{NP}}^{e^{+} e^{-} \rightarrow h h Z}$ & $\Delta r_{\mathrm{NP}}^{\gamma \gamma \rightarrow h h}$ & $\Delta r_{\mathrm{NP}}^{e^{+} e^{-} \rightarrow h h \nu \bar{\nu}}$ \\
\hline \hline THDM & 120 & $+120 \%$ & $-50 \%$ & $+(80-70) \%$ & $+50 \%$ & $-(80-50) \%$ \\
\hline $\mathrm{THDM}$ & 160 & $+70 \%$ & $-50 \%$ & $+(60-50) \%$ & $+110 \%$ & $-(80-50) \%$ \\
\hline $\mathrm{LQ}(Q=1 / 3,4 / 3)$ & 120 & $+150 \%$ & $-40 \%$ & $+(110-100) \%$ & $+130 \%,+100 \%$ & $-(70-60) \%$ \\
\hline $\mathrm{LQ}(Q=1 / 3,4 / 3)$ & 185 & $+60 \%$ & $-30 \%$ & $+50 \%$ & $+150 \%,+150 \%$ & $-(80-50) \%$ \\
\hline $\mathrm{Ch} 4$ & 120 & $-590 \%$ & $+7800 \%$ & $-(30-20) \%$ & $+3100 \%$ & $+(260-110) \%$ \\
\hline $\mathrm{Ch} 4$ & 210 & $-140 \%$ & $+2200 \%$ & - & - & $+(970-210) \%$ \\
\hline $\mathrm{Vec}$ & 120 & $-4 \%$ & $-10 \%$ & $-2 \%$ & $-10 \%$ & $+(5-1) \%$ \\
\hline Vec & 160 & $-2 \%$ & $-5 \%$ & $-1 \%$ & $-10 \%$ & $+(3-0) \%$ \\
\hline
\end{tabular}

TABLE III: Possible quantum corrections to the $h h h$ coupling constant, $\frac{\Gamma_{h h h}^{\mathrm{NP}}\left(4 m_{h}^{2}, m_{h}^{2}, m_{h}^{2}\right)}{\Gamma_{h h h}^{\mathrm{SM}}\left(4 m_{h}^{2}, m_{h}^{2}, m_{h}^{2}\right)}-1$, and deviations of cross sections $\Delta r_{\mathrm{NP}} \equiv\left(\sigma_{\mathrm{NP}}-\sigma_{\mathrm{SM}}\right) / \sigma_{\mathrm{SM}}$ are listed. The proton-proton collision energy is taken as $\sqrt{s}=14 \mathrm{TeV}$ for the gluon fusion mechanism. For the double-Higgs-strahlung $\sqrt{s_{e e}}$ is varied from $400 \mathrm{GeV}$ to $500 \mathrm{GeV}$, while for the $W$ boson fusion $\sqrt{s_{e e}}$ is varied from $1 \mathrm{TeV}$ to 3 TeV. For photon-photon collisions $\sqrt{s_{e e}}$ is optimized to obtain the largest cross sections. Model parameters are chosen as $m_{\Phi}=400 \mathrm{GeV}$ and $M^{2}=0$ for THDM, $m_{\phi}=400 \mathrm{GeV}$ and $M_{L \mathrm{Q}}^{2}=0$ for scalar leptoquark models, $m_{t^{\prime}}=400 \mathrm{GeV}$ and $m_{b^{\prime}}=345 \mathrm{GeV}$ for the fourth generation model, and $Y_{T}=1, M_{T}=1100 \mathrm{GeV}$ for vectorlike top-quark model, respectively.

A qualitative difference from the THDM case is that the scalar leptoquarks are colored, which can contribute to the $g g \rightarrow h h$ through the one particle irreducible one-loop diagram. However, it turns out that the top-quark one-loop contribution is much larger than the leptoquark-loop contribution, so that the SM result with $\Delta \kappa$ correction is a good approximation. Therefore, as expected in the analysis by using the $\Delta \kappa$, positive deviations in the hh h coupling constant make the cross sections smaller. It amounts to minus $40(30) \%$ for $m_{h}=120(185) \mathrm{GeV}$ assuming the other parameters as $m_{\phi}=400 \mathrm{GeV}$ and $M_{\mathrm{IQ}}^{2}=0$. The production rates for $e^{+} e^{-} \rightarrow h h Z$ can be enhanced by $+100(+50) \%$ for $m_{h}=120(185) \mathrm{GeV}$ due to the constructive interference in the contribution from the diagram with a positively deviated hhh coupling constant and the other diagrams. On the contrary, cross sections for $e^{+} e^{-} \rightarrow h h \nu \bar{\nu}$ become smaller due to the destructive interference. The production rates for $\gamma \gamma \rightarrow h h$ depend on electric charges of leptoquarks. For the scalar leptoquark with $Q=4 / 3$, the cross section can be enhanced by the threshold effect at $\sqrt{s_{\gamma \gamma}} \sim 2 m_{\phi}$, while for those with $Q=1 / 3$ such effects are smeared. For $\sqrt{s_{e e}} \gtrsim 500 \mathrm{GeV}$, the cross section for the scalar leptoquarks with $Q=4 / 3$ can enhance more than several times $+100 \%$.

In the model with chiral fourth generation quarks, the $h$ hh coupling constant can be changed by more than a few times $-100 \%$ due to the nondecoupling loop effect of additional heavy chiral fermions. These huge corrections can be possible under the constraint from the data for precision measurements and the direct search results. However, such a large fermionic loop contribution can make Higgs potential unstable, so that a heavier Higgs boson is required than the allowed value in the SM to recover the stability of vacuum. 
A light Higgs boson can be allowed by extending the Higgs sector with additional scalar doublets. The cross section for $g g \rightarrow h h$ is drastically enhanced from the SM prediction by a factor of 10-100, because new colored particles contribute to the additional one-loop diagrams at leading order and the fourth generation fermions enhance both the one-loop induced vertex $g g h$ and the one-loop corrected $h h h$ coupling constant. For a reference point of $m_{t^{\prime}}=m_{\ell}=400 \mathrm{GeV}$ and $m_{b^{\prime}}=m_{\nu^{\prime}}=345 \mathrm{GeV}$, the cross sections become 7800 $(2200) \%$ for $m_{h}=120(210) \mathrm{GeV}$. Consequently, the process $g g \rightarrow h h$ with the decay mode of $h \rightarrow W W / Z Z$ can be promising to measure the $h h h$ coupling constant for $m_{h} \gtrsim 210$ $\mathrm{GeV}$. For $e^{+} e^{-} \rightarrow h h Z$ process, the effect of fourth generation fermions only appear in the one-loop corrected hhh coupling constant. Since this correction is negative because of the fermionic loop contribution, the cross section is suppressed. Numerically, it is reduced by $30-20 \%$ for the above reference point with $m_{h}=120 \mathrm{GeV}$ and $400 \mathrm{GeV} \lesssim \sqrt{s_{e e}} \lesssim 500$ $\mathrm{GeV}$. On the contrary, in $e^{+} e^{-} \rightarrow h h \nu \bar{\nu}$ process, the effect of fourth generation fermions only appears in the $h h h$ coupling constant through large one-loop corrections, which makes the cross section huge as compared to the SM value. Cross sections for $\gamma \gamma \rightarrow h h$ at photon colliders can also be modified by extra fermion loops similarly to those for $g g \rightarrow h h$. With an optimized value of $\sqrt{s_{e e}} \lesssim 500 \mathrm{GeV}$, the cross section can be enhanced by $3100 \%$ for the reference point with $m_{h}=120 \mathrm{GeV}$. Since the cross sections are drastically enhanced for $m_{h}=120 \mathrm{GeV}$ and hadronic decay modes can be measurable at the PLC, the process $\gamma \gamma \rightarrow h h$ would be a promising process to probe the $h h h$ coupling constant.

In the vectorlike quark model, a nondecoupling limit $\left(M_{T} \simeq 0\right)$ cannot be taken due to the severe experimental constraints. Therefore, there are no large one-loop effect from a vector top-quark on the $h h h$ coupling constant. The cross sections for $g g \rightarrow h h$ and $\gamma \gamma \rightarrow h h$ can be deviated slightly from SM prediction due to the flavor changing Yukawa interaction of vectorlike quarks. However, deviations of Higgs boson pair production cross sections are rather small, so that huge luminosity would be required for measuring the deviation of the hhh coupling constant.

Measuring four kinds of double Higgs boson production processes at different future collider experiments is useful to discriminate whether new physics particles in the loop are fermions or bosons and also whether they are colored or not. Higgs boson pair production processes $e^{+} e^{-} \rightarrow h h Z$ and $e^{+} e^{-} \rightarrow h h \nu \bar{\nu}$ at lepton colliders are tree-level processes, which enable us to extract information of the $h h h$ coupling constant. On the other hand, measurements of effective vertices $g g h h$ and $\gamma \gamma h h$ in loop induced processes $g g \rightarrow h h$ at the LHC and $\gamma \gamma \rightarrow h h$ at the PLC can provide information of colored and electrically charged particles in loop diagrams. Effective vertices $g g h$ and $\gamma \gamma h$ can be determined in the single Higgs boson production processes $g g \rightarrow h$ and $\gamma \gamma \rightarrow h$ as well. Combining these measurements, we would be able to disentangle new physics effects in the $h h h$ coupling and the effective vertices.

We have considered the Higgs boson pair production processes, $g g \rightarrow h h, e^{+} e^{-} \rightarrow h h Z$, $e^{+} e^{-} \rightarrow h h \nu \bar{\nu}$ and $\gamma \gamma \rightarrow h h$ as a probe of the $h h h$ coupling constant. The measurement of the $h h h$ coupling constant is particularly important to understand the mechanism of the electroweak symmetry breaking. The $h h h$ coupling constant can receive quite large quantum 
corrections from new physics particles as a nondecoupling effect, which can be an order of more than 100\%. Deviations of the hhh coupling constant can give different effects on these processes which can largely modify production cross sections. Additional particles in new physics model can also significantly affect the $g g \rightarrow h h$ and the $\gamma \gamma \rightarrow h h$ processes according to their color and electric charges. We have found that these four Higgs boson pair production processes at different colliders can play complementary roles in exploring new physics through the Higgs sector.

\section{Acknowledgments}

We would like to thank Kaoru Hagiwara for useful comments. S. K. acknowledges support from Grant-in-Aid for Science Research, Japan Society for the Promotion of Science (JSPS), No. 18034004. Y. O. acknowledges support from Grant-in-Aid for Science Research, MEXTJapan, No. 16081211, and JSPS, No. 20244037. S. K. and Y. O. acknowledge support from Grant-in-Aid for Scientific Research No. 22244031 of JSPS. Numerical calculations were partly carried out on Altix3700 at Yukawa Institute for Theoretical Physics (YITP) in Kyoto University.

\section{Appendix A: The one-loop corrections to the triple Higgs boson coupling}

The relatively large one-loop correction to the $h h h$ coupling constant in the SM has been calculated as [7],

$$
\begin{aligned}
\frac{\Gamma_{h h h}^{\mathrm{SM}}\left(p_{1}^{2}, p_{2}^{2}, q^{2}\right)}{\lambda_{h h h}^{\mathrm{SM}}}=1-\frac{N_{c}}{16 \pi^{2}}\left\{+\sum_{f=t, b} \frac{m_{f}^{2}}{m_{h}^{2} v^{2}}\left(-2 m_{h}^{2}+8 m_{f}^{2}\right) B_{0}\left(m_{h}^{2} ; m_{f}, m_{f}\right)\right. & -\sum_{\left(f_{1}, f_{2}\right)=(t, b)} \frac{4}{v^{2}}\left[B_{22}\left(0 ; m_{f_{1}}, m_{f_{2}}\right)-\frac{1}{4}\left(m_{f_{1}}^{2}+m_{f_{2}}^{2}\right)\right] \\
+ & \sum_{f=t, b} \frac{3 m_{f}^{2}}{2 v^{2}}\left[2 B_{0}\left(m_{h}^{2} ; m_{f}, m_{f}\right)-\left(-2 m_{h}^{2}+8 m_{f}^{2}\right) B_{0}^{\prime}\left(m_{h}^{2} ; m_{f}, m_{f}\right)\right] \\
& -\sum_{f=t, b} \frac{8 m_{f}^{4}}{3 m_{h}^{2} v^{2}}\left[B_{0}\left(p_{1}^{2} ; m_{f}, m_{f}\right)+B_{0}\left(p_{2}^{2} ; m_{f}, m_{f}\right)+B_{0}\left(q^{2} ; m_{f}, m_{f}\right)\right. \\
& \left.\left.-\frac{1}{2}\left(p_{1}^{2}+p_{2}^{2}+q^{2}-8 m_{f}^{2}\right) C_{0}\left(p_{1}^{2}, p_{2}^{2}, q^{2} ; m_{f}, m_{f}, m_{f}\right)\right]\right\}
\end{aligned}
$$

where $B$ and $C$ are the loop functions, which are defined in Ref. [73]. For the chiral fourth generation model, all the extra fermions further contribute to the $h h h$ coupling constant. Then the sum should be replaced by all heavy fermions in the preceding formula.

In the leptoquark model, the quantum effect is given by

$\frac{\Gamma_{h h h}^{\mathrm{LQ}}\left(p_{1}^{2}, p_{2}^{2}, q^{2}\right)}{\lambda_{h h h}^{\mathrm{SM}}}=\frac{\Gamma_{h h h}^{\mathrm{SM}}\left(p_{1}^{2}, p_{2}^{2}, q^{2}\right)}{\lambda_{h h h}^{\mathrm{SM}}}+\frac{N_{c}}{16 \pi^{2}}\left\{+\frac{1}{m_{h}^{2}} \lambda_{h \phi \phi^{*}}^{2} B_{0}\left(m_{h}^{2} ; m_{\phi}, m_{\phi}\right)-\frac{3}{2} \lambda_{h \phi \phi^{*}}^{2} B_{0}^{\prime}\left(m_{h}^{2} ; m_{\phi}, m_{\phi}\right)\right.$ 


$$
\begin{aligned}
& -\frac{v}{3 m_{h}^{2}}\left[-2 \lambda_{h \phi \phi^{*}}^{3} C_{0}\left(p_{1}^{2}, p_{2}^{2}, q^{2} ; m_{\phi}, m_{\phi}, m_{\phi}\right)\right. \\
& \left.\left.\quad+2 \lambda_{h \phi \phi^{*}} \frac{\lambda_{h h \phi \phi^{*}}}{v}\left[B_{0}\left(q^{2} ; m_{\phi}, m_{\phi}\right)+B_{0}\left(p_{1}^{2} ; m_{\phi}, m_{\phi}\right)+B_{0}\left(p_{2}^{2} ; m_{\phi}, m_{\phi}\right)\right]\right]\right\} .
\end{aligned}
$$

In the THDM with the SM-like limit $\sin (\beta-\alpha)=1$, the one-loop corrections to the $h h h$ coupling constant are calculated as [7],

$$
\begin{aligned}
& \frac{\Gamma_{h h h}^{\mathrm{THDM}}\left(p_{1}^{2}, p_{2}^{2}, q^{2}\right)}{\lambda_{h h h}^{\mathrm{SM}}}=\frac{\Gamma_{h h h}^{\mathrm{SM}}\left(p_{1}^{2}, p_{2}^{2}, q^{2}\right)}{\lambda_{h h h}^{\mathrm{SM}}}+\frac{1}{16 \pi^{2}}\{ \\
& +\frac{1}{m_{h}^{2}}\left[\lambda_{h H^{+} H^{-}}^{2} B_{0}\left(m_{h}^{2} ; m_{H^{ \pm}}, m_{H^{ \pm}}\right)+2 \lambda_{h H H}^{2} B_{0}\left(m_{h}^{2} ; m_{H}, m_{H}\right)+2 \lambda_{h A A}^{2} B_{0}\left(m_{h}^{2} ; m_{A}, m_{A}\right)\right] \\
& -\frac{1}{2 v^{2}}\left[B_{5}\left(0 ; m_{H}, m_{H^{ \pm}}\right)+B_{5}\left(0 ; m_{A}, m_{H^{ \pm}}\right)\right] \\
& -\frac{3}{2}\left[\lambda_{h H^{+} H^{-}}^{2} B_{0}^{\prime}\left(m_{h}^{2} ; m_{H^{ \pm}}, m_{H^{ \pm}}\right)+2 \lambda_{h H H}^{2} B_{0}^{\prime}\left(m_{h}^{2} ; m_{H}, m_{H}\right)+2 \lambda_{h A A}^{2} B_{0}^{\prime}\left(m_{h}^{2} ; m_{A}, m_{A}\right)\right] \\
& -\frac{v}{3 m_{h}^{2}}\left[-2 \lambda_{h H^{+} H^{-}}^{3} C_{0}\left(p_{1}^{2}, p_{2}^{2}, q^{2} ; m_{H^{ \pm}}, m_{H^{ \pm}}, m_{H^{ \pm}}\right)\right. \\
& \quad-8 \lambda_{h H H^{3}}^{3} C_{0}\left(p_{1}^{2}, p_{2}^{2}, q^{2} ; m_{H}, m_{H}, m_{H}\right)-8 \lambda_{h A A}^{3} C_{0}\left(p_{1}^{2}, p_{2}^{2}, q^{2} ; m_{A}, m_{A}, m_{A}\right) \\
& \quad+2 \lambda_{h H^{+} H^{-}} \frac{\lambda_{h h H^{+} H^{-}}}{v}\left[B_{0}\left(q^{2} ; m_{H^{ \pm}}, m_{H^{ \pm}}\right)+B_{0}\left(p_{1}^{2} ; m_{H^{ \pm}}, m_{H^{ \pm}}\right)+B_{0}\left(p_{2}^{2} ; m_{H^{ \pm}}, m_{H^{ \pm}}\right)\right] \\
& \quad+4 \lambda_{h H H} \frac{\lambda_{h h H H}}{v}\left[B_{0}\left(q^{2} ; m_{H}, m_{H}\right)+B_{0}\left(p_{1}^{2} ; m_{H}, m_{H}\right)+B_{0}\left(p_{2}^{2} ; m_{H}, m_{H}\right)\right] \\
& \left.\quad+4 \lambda_{h A A} \frac{\lambda_{h h A A}}{v}\left[B_{0}\left(q^{2} ; m_{A}, m_{A}\right)+B_{0}\left(p_{1}^{2} ; m_{A}, m_{A}\right)+B_{0}\left(p_{2}^{2} ; m_{A}, m_{A}\right)\right]\right\}
\end{aligned}
$$

where

$$
\begin{gathered}
\lambda_{h H^{+} H^{-}}=2 \lambda_{h h H^{+} H^{-}}=-\frac{m_{h}^{2}}{v}-\frac{2 m_{H^{ \pm}}^{2}}{v}\left(1-\frac{M^{2}}{m_{H^{ \pm}}^{2}}\right), \\
\lambda_{h H H}=2 \lambda_{h h H H}=\frac{1}{2}\left[-\frac{m_{h}^{2}}{v}-\frac{2 m_{H}^{2}}{v}\left(1-\frac{M^{2}}{m_{H}^{2}}\right)\right], \\
\lambda_{h A A}=2 \lambda_{h h A A}=\frac{1}{2}\left[-\frac{m_{h}^{2}}{v}-\frac{2 m_{A}^{2}}{v}\left(1-\frac{M^{2}}{m_{A}^{2}}\right)\right] .
\end{gathered}
$$

In the vectorlike top-quark model, the one-loop corrected $h$ hh coupling constant is given by

$$
\begin{aligned}
& \frac{\Gamma_{h h h}^{\mathrm{Vec}}\left(p_{1}^{2}, p_{2}^{2}, q^{2}\right)}{\lambda_{h h h}^{\mathrm{SM}}}=1-\frac{N_{c}}{16 \pi^{2}}\{ \\
& +\frac{1}{m_{h}^{2}}\left\{-4 \frac{m_{f}}{v}\left(\frac{y_{t}^{\mathrm{eff}}}{\sqrt{2}}\right) A\left(m_{t}\right)+\left(\frac{y_{t}^{\mathrm{eff}}}{\sqrt{2}}\right)^{2}\left[4 A\left(m_{t}\right)+\left(-2 m_{h}^{2}+8 m_{t}^{2}\right) B_{0}\left(m_{h}^{2} ; m_{t}, m_{t}\right)\right]\right.
\end{aligned}
$$




$$
\begin{aligned}
& -4 \frac{m_{T}}{v}\left(\frac{y_{T}^{\mathrm{eff}}}{\sqrt{2}}\right) A\left(m_{T}\right)+\left(\frac{y_{T}^{\mathrm{eff}}}{\sqrt{2}}\right)^{2}\left[4 A\left(m_{T}\right)+\left(-2 m_{h}^{2}+8 m_{T}^{2}\right) B_{0}\left(m_{h}^{2} ; m_{T}, m_{T}\right)\right] \\
& +2\left[\left(\frac{\epsilon_{t}}{\sqrt{2}}\right)^{2}+\left(\frac{\epsilon_{T}}{\sqrt{2}}\right)^{2}\right]\left[A\left(m_{t}\right)+A\left(m_{T}\right)+\left(-m_{h}^{2}+m_{t}^{2}+m_{T}^{2}\right) B_{0}\left(m_{h}^{2} ; m_{t}, m_{T}\right)\right] \\
& \left.+8\left(\frac{\epsilon_{t}}{\sqrt{2}}\right)\left(\frac{\epsilon_{T}}{\sqrt{2}}\right) m_{t} m_{T} B_{0}\left(m_{h}^{2} ; m_{t}, m_{T}\right)\right\} \\
& -\frac{1}{v^{2}}\left[4 c_{L}^{2} B_{22}\left(0 ; m_{t}, 0\right)-c_{L}^{2} m_{t}^{2}+4 s_{L}^{2} B_{22}\left(0 ; m_{T}, 0\right)-s_{L}^{2} m_{T}^{2}\right] \\
& +\frac{3}{2}\left\{+\left(\frac{y_{t}^{\mathrm{eff}}}{\sqrt{2}}\right)^{2}\left[2 B_{0}\left(m_{h}^{2} ; m_{t}, m_{t}\right)-\left(-2 m_{h}^{2}+8 m_{t}^{2}\right) B_{0}^{\prime}\left(m_{h}^{2} ; m_{t}, m_{t}\right)\right]\right. \\
& +\left(\frac{y_{T}^{\mathrm{eff}}}{\sqrt{2}}\right)^{2}\left[2 B_{0}\left(m_{h}^{2} ; m_{T}, m_{T}\right)-\left(-2 m_{h}^{2}+8 m_{T}^{2}\right) B_{0}^{\prime}\left(m_{h}^{2} ; m_{T}, m_{T}\right)\right] \\
& +2\left[\left(\frac{\epsilon_{t}}{\sqrt{2}}\right)^{2}+\left(\frac{\epsilon_{T}}{\sqrt{2}}\right)^{2}\right]\left[B_{0}\left(m_{h}^{2} ; m_{t}, m_{T}\right)-\left(-m_{h}^{2}+m_{t}^{2}+m_{T}^{2}\right) B_{0}^{\prime}\left(m_{h}^{2} ; m_{t}, m_{T}\right)\right] \\
& \left.-8\left(\frac{\epsilon_{t}}{\sqrt{2}}\right)\left(\frac{\epsilon_{T}}{\sqrt{2}}\right) m_{t} m_{T} B_{0}^{\prime}\left(m_{h}^{2} ; m_{t}, m_{T}\right)\right\} \\
& -\frac{v}{3 m_{h}^{2}}\left\{8 m _ { t } ( \frac { y _ { t } ^ { \text { eff } } } { \sqrt { 2 } } ) ^ { 3 } \left[+B_{0}\left(p_{1}^{2} ; m_{t}, m_{t}\right)+B_{0}\left(p_{2}^{2} ; m_{t}, m_{t}\right)+B_{0}\left(q^{2} ; m_{t}, m_{t}\right)\right.\right. \\
& \left.-\frac{1}{2}\left(p_{1}^{2}+p_{2}^{2}+q^{2}-8 m_{t}^{2}\right) C_{0}\left(p_{1}^{2}, p_{2}^{2}, q^{2} ; m_{t}, m_{t}, m_{t}\right)\right] \\
& +4 m_{t}\left(\frac{y_{t}^{\text {eff }}}{\sqrt{2}}\right)\left[\left(\frac{\epsilon_{t}}{\sqrt{2}}\right)^{2}+\left(\frac{\epsilon_{T}}{\sqrt{2}}\right)^{2}\right] \\
& \times\left[+B_{0}\left(p_{1}^{2} ; m_{t}, m_{t}\right)+B_{0}\left(p_{2}^{2} ; m_{t}, m_{t}\right)+B_{0}\left(q^{2} ; m_{t}, m_{t}\right)\right. \\
& +B_{0}\left(p_{1}^{2} ; m_{t}, m_{T}\right)+B_{0}\left(p_{2}^{2} ; m_{t}, m_{T}\right)+B_{0}\left(q^{2} ; m_{t}, m_{T}\right) \\
& -\frac{1}{2}\left(p_{1}^{2}+p_{2}^{2}-2 m_{t}^{2}-2 m_{T}^{2}\right) C_{0}\left(p_{1}^{2}, p_{2}^{2}, q^{2} ; m_{t}, m_{T}, m_{t}\right) \\
& -\frac{1}{2}\left(p_{1}^{2}+q^{2}-2 m_{t}^{2}-2 m_{T}^{2}\right) C_{0}\left(p_{1}^{2}, p_{2}^{2}, q^{2} ; m_{T}, m_{t}, m_{t}\right) \\
& \left.-\frac{1}{2}\left(p_{2}^{2}+q^{2}-2 m_{t}^{2}-2 m_{T}^{2}\right) C_{0}\left(p_{1}^{2}, p_{2}^{2}, q^{2} ; m_{t}, m_{t}, m_{T}\right)\right] \\
& +8 m_{T}\left(\frac{y_{t}^{\mathrm{eff}}}{\sqrt{2}}\right)\left(\frac{\epsilon_{t}}{\sqrt{2}}\right)\left(\frac{\epsilon_{T}}{\sqrt{2}}\right) \\
& \times\left[+B_{0}\left(p_{1}^{2} ; m_{t}, m_{T}\right)+B_{0}\left(p_{2}^{2} ; m_{t}, m_{T}\right)+B_{0}\left(q^{2} ; m_{t}, m_{T}\right)\right. \\
& -\frac{1}{2}\left(q^{2}-4 m_{t}^{2}\right) C_{0}\left(p_{1}^{2}, p_{2}^{2}, q^{2} ; m_{t}, m_{T}, m_{t}\right) \\
& -\frac{1}{2}\left(p_{2}^{2}-4 m_{t}^{2}\right) C_{0}\left(p_{1}^{2}, p_{2}^{2}, q^{2} ; m_{T}, m_{t}, m_{t}\right) \\
& \left.-\frac{1}{2}\left(p_{1}^{2}-4 m_{t}^{2}\right) C_{0}\left(p_{1}^{2}, p_{2}^{2}, q^{2} ; m_{t}, m_{t}, m_{T}\right)\right]
\end{aligned}
$$




$$
\left.\left.+\left(y_{t}^{\mathrm{eff}} \rightarrow y_{T}^{\mathrm{eff}}, m_{t} \leftrightarrow m_{T}\right)\right\}\right\}
$$

where

$$
\begin{aligned}
y_{t}^{\mathrm{eff}} & =c_{L}\left(c_{R} y_{t}-s_{R} Y_{T}\right), \\
\epsilon_{t} & =s_{L}\left(c_{R} y_{t}-s_{R} Y_{T}\right), \\
y_{T}^{\mathrm{eff}} & =s_{L}\left(s_{R} y_{t}+c_{R} Y_{T}\right), \\
\epsilon_{T} & =c_{L}\left(s_{R} y_{t}+c_{R} Y_{T}\right) .
\end{aligned}
$$

\section{Appendix B: The loop integrals for $g g \rightarrow h h$ and $\gamma \gamma \rightarrow h h$}

The SM contributions to the loop functions in $g g \rightarrow h h$ and $\gamma \gamma \rightarrow h h$ amplitudes can be found in Refs. [21, 29]. It can be generalized straightforwardly for the chiral fourth generation model.

In leptoquark models, in addition to the SM fermions there are contributions from the colored scalar particle to $g g \rightarrow h h$ amplitude as,

$$
\begin{aligned}
F_{\triangle}^{\phi}= & \frac{4 \lambda_{h h \phi \phi^{*}}}{v}\left(1-\frac{\hat{s}-m_{h}^{2}}{\lambda_{h h h} v}\right)\left(1+2 m_{\phi}^{2} C_{0}^{(1,2)}\right), \\
F_{\square}^{\phi}=+4 \lambda_{h \phi \phi^{*}}^{2}\left\{m_{\phi}^{2}\left(D_{0}^{(1,2,3)}+D_{0}^{(2,1,3)}+D_{0}^{(1,3,2)}\right)\right. & \\
& \left.+\frac{\hat{t} \hat{u}-m_{h}^{4}}{2 \hat{s}} D_{0}^{(1,3,2)}-\frac{\hat{u}-m_{h}^{2}}{\hat{s}} C_{0}^{(2,3)}-\frac{\hat{t}-m_{h}^{2}}{\hat{s}} C_{0}^{(1,3)}\right\}, \\
G_{\square}^{\phi}=+ & 4 \lambda_{h \phi \phi^{*}}^{2}\left\{-C_{0}^{(3,4)}+m_{\phi}^{2}\left(D_{0}^{(1,2,3)}+D_{0}^{(2,1,3)}+D_{0}^{(1,3,2)}\right)\right. \\
& +\frac{1}{2\left(\hat{t} \hat{u}-m_{h}^{4}\right)}\left[-2 \hat{u}\left(\hat{u}-m_{h}^{2}\right) C_{0}^{(2,3)}-2 \hat{t}\left(\hat{t}-m_{h}^{2}\right) C_{0}^{(1,3)}\right. \\
& \left.\left.+\hat{s} \hat{u}^{2} D_{0}^{(1,2,3)}+\hat{s} \hat{t}^{2} D_{0}^{(2,1,3)}+\hat{s}\left(\hat{s}-2 m_{h}^{2}\right) C_{0}^{(1,2)}+\hat{s}\left(\hat{s}-4 m_{h}^{2}\right) C_{0}^{3,4}\right]\right\},
\end{aligned}
$$

where

$$
\lambda_{h \phi \phi^{*}}=2 \lambda_{h h \phi \phi^{*}}=-\frac{2 m_{\phi}^{2}}{v}\left(1-\frac{M_{L \mathrm{Q}}^{2}}{m_{\phi}^{2}}\right),
$$

and the loop functions are abbreviated as

$$
\begin{aligned}
& C_{A[i, j, k]}^{(1,2)}=C_{A}\left(0,0, \hat{s} ; m_{i}, m_{j}, m_{k}\right), \\
& C_{A[i, j, k]}^{(3,4)}=C_{A}\left(\hat{s}, m_{h}^{2}, m_{h}^{2} ; m_{i}, m_{j}, m_{k}\right),
\end{aligned}
$$




$$
\begin{aligned}
C_{A[i, j, k]}^{(1,3)} & =C_{A}\left(\hat{t}, 0, m_{h}^{2} ; m_{i}, m_{j}, m_{k}\right), \\
C_{A[i, j, k]}^{(2,3)} & =C_{A}\left(m_{h}^{2}, 0, \hat{u} ; m_{i}, m_{j}, m_{k}\right), \\
D_{A[i, j, k, l]}^{(1,2,3)} & =D_{A}\left(0,0, m_{h}^{2}, m_{h}^{2}, \hat{s}, \hat{u} ; m_{i}, m_{j}, m_{k}, m_{l}\right), \\
D_{A[i, j, k, l]}^{(2,1,3)} & =D_{A}\left(0,0, m_{h}^{2}, m_{h}^{2}, \hat{s}, \hat{t} ; m_{i}, m_{j}, m_{k}, m_{l}\right), \\
D_{A[i, j, k, l]}^{(1,3,2)} & =D_{A}\left(0, m_{h}^{2}, 0, m_{h}^{2}, \hat{t}, \hat{u} ; m_{i}, m_{j}, m_{k}, m_{l}\right) .
\end{aligned}
$$

The index $A$ denotes classes of loop functions, and we omitted obvious mass indices of intermediate particles in the Eqs.(B1)-(B33). The leptoquarks also contribute to $\gamma \gamma \rightarrow h h$ process. These additional contributions to the amplitude are basically the same as those loop integrals for $g g \rightarrow h h$ amplitude except for electric charges and a color factor; $H_{\phi}^{++}=$ $N_{c}^{\phi} Q_{\phi}^{2} F^{\phi}$ and $H_{\phi}^{+-}=N_{c}^{\phi} Q_{\phi}^{2} G^{\phi}$.

The SM contribution can be modified in the vectorlike quark model because of $t$ and $T$ mixing. The vectorlike top-quark $T$ also gives additional contributions to $g g \rightarrow h h$ and $\gamma \gamma \rightarrow h h$ amplitudes as

$$
\begin{aligned}
& F_{\triangle}^{\mathrm{Vec}}=2\left(\frac{y_{t}^{\mathrm{eff}}}{\sqrt{2}}\right)\left(\frac{m_{t}}{v}\right)\left[-4 B_{0}^{(1+2)}+16 C_{24}^{(1,2)}-2 \hat{s} C_{0}^{(1,2)}\right]_{t}+\left(y_{t}^{\mathrm{eff}} \rightarrow y_{T}^{\mathrm{eff}}, m_{t} \leftrightarrow m_{T}\right), \\
& F_{\square}^{\mathrm{Vec}}+G_{\square}^{\mathrm{Vec}}=-2\left(\frac{y_{t}^{\mathrm{eff}}}{\sqrt{2}}\right)^{2}\left\{-4 B_{0}^{(1+2)}+16 C_{24}^{(1,2)}-2 \hat{s} C_{0}^{(1,2)}\right. \\
& +\hat{s}\left[\left(\hat{u}-4 m_{t}^{2}\right) D_{0}^{(1,2,3)}+\left(\hat{t}-4 m_{t}^{2}\right) D_{0}^{(2,1,3)}\right] \\
& -\left[\left(\hat{t}-m_{h}^{2}\right)\left(\hat{u}-m_{h}^{2}\right)+\hat{s}\left(4 m_{t}^{2}-m_{h}^{2}\right)\right] D_{0}^{(1,3,2)} \\
& \left.+4\left(\hat{s}-2 m_{h}^{2}+8 m_{t}^{2}\right)\left[D_{27}^{(1,2,3)}+D_{27}^{(2,1,3)}+D_{27}^{(1,3,2)}-\frac{1}{2} C_{0}^{(3,4)}\right]\right\}_{t} \\
& -\left[\left(\frac{\epsilon_{t}}{\sqrt{2}}\right)^{2}+\left(\frac{\epsilon_{T}}{\sqrt{2}}\right)^{2}\right]\left\{-4 B_{0[t, t]}^{(1+2)}+16 C_{24[t, t, t]}^{(1,2)}-2 \hat{s} C_{0[t, t, t]}^{(1,2)}\right. \\
& +\hat{s}\left[\left(\hat{u}-m_{t}^{2}-m_{T}^{2}\right) D_{0[t, t, t, T]}^{(1,2,3)}+\left(\hat{t}-m_{t}^{2}-m_{T}^{2}\right) D_{0[t, t, t, T]}^{(2,1,3)}\right] \\
& -\left[\left(\hat{t}-m_{h}^{2}\right)\left(\hat{u}-m_{h}^{2}\right)+\hat{s}\left(m_{t}^{2}+m_{T}^{2}-m_{h}^{2}\right)\right] D_{0[t, T, t, T]}^{(1,3,2)} \\
& \left.+4\left(\hat{s}-2 m_{h}^{2}+2 m_{t}^{2}+2 m_{T}^{2}\right)\left(D_{27[t, t, t, T]}^{(1,2,3)}+D_{27[t, t, t, T]}^{(2,1,3)}+D_{27[t, T, t, T]}^{(1,3,2)}-\frac{1}{2} C_{0[t, t, T]}^{(3,4)}\right)\right\} \\
& +4\left(\frac{\epsilon_{t}}{\sqrt{2}}\right)\left(\frac{\epsilon_{T}}{\sqrt{2}}\right) m_{t} m_{T}\left\{\hat{s}\left(D_{0[t, t, t, T]}^{(1,2,3)}+D_{0[t, t, t, T]}^{(2,1,3)}+D_{0[t, T, t, T]}^{(1,3,2)}\right)\right. \\
& \left.-4\left(D_{27[t, t, t, T]}^{(1,2,3)}+D_{27[t, t, t, T]}^{(2,1,3)}+D_{27[t, T, t, T]}^{(1,3,2)}-C_{0[t, t, T]}^{(3,4)}\right)\right\} \\
& +\left(y_{t}^{\mathrm{eff}} \rightarrow y_{T}^{\mathrm{eff}}, m_{t} \leftrightarrow m_{T}\right) \\
& G_{\square}^{\mathrm{Vec}}=-\frac{\hat{t} \hat{u}-m_{h}^{4}}{2 \hat{s}}\left\{-8\left(\frac{y_{t}^{\mathrm{eff}}}{\sqrt{2}}\right)^{2}\left[\left(\hat{s}-2 m_{h}^{2}+8 m_{t}^{2}\right)\left[D_{23}^{(1,2,3)}+D_{23}^{(2,1,3)}+D_{12}^{(1,3,2)}+D_{22}^{(1,3,2)}\right]\right.\right.
\end{aligned}
$$




$$
\begin{gathered}
\left.+\hat{s}\left(D_{13}^{(1,2,3)}+D_{13}^{(2,1,3)}\right)-\left(C_{0}^{(1,3)}+C_{0}^{(2,3)}+C_{11}^{(1,3)}+C_{11}^{(2,3)}+\bar{C}_{12}^{(1,3)}+\bar{C}_{12}^{(2,3)}\right)\right]_{t} \\
+4\left[\left(\frac{\epsilon_{t}}{\sqrt{2}}\right)^{2}+\left(\frac{\epsilon_{T}}{\sqrt{2}}\right)^{2}\right]\left[+\hat{s}\left(D_{13[t, t, t, T]}^{(1,2,3)}+D_{13[t, t, t, T]}^{(2,1,3)}\right)\right. \\
+\left(\hat{s}-2 m_{h}^{2}+2 m_{t}^{2}+2 m_{T}^{2}\right)\left(D_{23[t, t, t, T]}^{(1,2,3)}+D_{23[t, t, t, T]}^{(2,1,3)}+D_{12[t, T, t, T]}^{(1,3,2)}+D_{22[t, T, t, T]}^{(1,3,2)}\right) \\
\left.-\left(C_{0[t, t, T]}^{(1,3)}+C_{0[t, t, T]}^{(2,3)}+C_{11[t, t, T]}^{(1,3)}+C_{11[t, t, T]}^{(2,3)}+\bar{C}_{12[t, t, T]}^{(1,3)}+\bar{C}_{12[t, t, T]}^{(2,3)}\right)\right] \\
\quad-16\left(\frac{\epsilon_{t}}{\sqrt{2}}\right)\left(\frac{\epsilon_{T}}{\sqrt{2}}\right) m_{t} m_{T}\left(D_{23[t, t, t, T]}^{(1,2,3)}+D_{23[t, t, t, T]}^{(2,1,3)}+D_{12[t, T, t, T]}^{(1,3,2)}+D_{22[t, T, t, T]}^{(1,3,2)}\right) \\
\}+\left(y_{t}^{\mathrm{eff}} \rightarrow y_{T}^{\mathrm{eff}}, m_{t} \leftrightarrow m_{T}\right),
\end{gathered}
$$

where

$$
\begin{aligned}
B_{A[i, j]}^{(1+2)} & =B_{A}\left(\hat{s} ; m_{i}, m_{j}\right), \\
\bar{C}_{A[i, j, k]}^{(1,3)} & =C_{A}\left(0, \hat{t}, m_{h}^{2} ; m_{i}, m_{j}, m_{k}\right), \\
\bar{C}_{A[i, j, k]}^{(2,3)} & =C_{A}\left(0, m_{h}^{2}, \hat{u} ; m_{i}, m_{j}, m_{k}\right) .
\end{aligned}
$$

The loop functions for photon collision are expressed as $H_{\mathrm{Vec}}^{++}=N_{c}^{t} Q_{t}^{2} F^{\mathrm{Vec}}$ and $H_{\mathrm{Vec}}^{+-}=$ $N_{c}^{t} Q_{t}^{2} G^{\mathrm{Vec}}$.

[1] R. Barate et al. [LEP Working Group for Higgs boson searches and ALEPH Collaboration and DELPHI Collaboration and L3 Collaboration and OPAL Collaboration], Phys. Lett. B 565, 61 (2003); S. Schael et al. [ALEPH Collaboration and DELPHI Collaboration and L3 Collaboration and OPAL Collaborations and LEP Working Group for Higgs Boson Searches], Eur. Phys. J. C 47, 547 (2006).

[2] The LEP Electroweak Working Group, http://lepewwg.web.cern.ch/LEPEWWG/

[3] T. Aaltonen et al. (CDF and D0 Collaborations), Phys. Rev. Lett. 104, 061802 (2010); T. Aaltonen et al. (CDF Collaboration), Phys. Rev. Lett. 104, 061803 (2010); V. M. Abazov et al. (D0 Collaboration), Phys. Rev. Lett. 104, 061804 (2010).

[4] New results presented by B. Kilminster at International Conference on High Energy Physics (ICHEP), summer 2010.

[5] T. Appelquist and J. Carazzone, Phys. Rev. D 11, 2856 (1975).

[6] D. C. Kennedy and B. W. Lynn, Nucl. Phys. B 322, 1 (1989); M. E. Peskin and T. Takeuchi, Phys. Rev. Lett. 65, 964 (1990), Phys. Rev. D 46, 381 (1992); G. Altarelli and R. Barbieri, Phys. Lett. B 253, 161 (1991).

[7] S. Kanemura, S. Kiyoura, Y. Okada, E. Senaha and C. P. Yuan, Phys. Lett. B 558, 157 (2003); S. Kanemura, Y. Okada, E. Senaha and C. P. Yuan, Phys. Rev. D 70, 115002 (2004). 
[8] C. Grojean, G. Servant and J. D. Wells, Phys. Rev. D 71, 036001 (2005).

[9] S. Kanemura, Y. Okada and E. Senaha, Phys. Lett. B 606, 361 (2005).

[10] S. W. Ham and S. K. Oh, arXiv:hep-ph/0502116.

[11] M. Aoki, S. Kanemura and O. Seto, Phys. Rev. Lett. 102, 051805 (2009), Phys. Rev. D 80, 033007 (2009).

[12] A. Noble and M. Perelstein, Phys. Rev. D 78, 063518 (2008).

[13] A. G. Cohen, D. B. Kaplan and A. E. Nelson, Ann. Rev. Nucl. Part. Sci. 43 (1993) 27; M. Quiros, Helv. Phys. Acta 67 (1994) 451; V. A. Rubakov and M. E. Shaposhnikov, Usp. Fiz. Nauk 166 (1996) 493 [Phys. Usp. 39 (1996) 461].

[14] G. D. Kribs, T. Plehn, M. Spannowsky and T. M. P. Tait, Phys. Rev. D 76, 075016 (2007).

[15] S. Kanemura, T. Shindou and K. Yagyu, arXiv:1009.1836 [hep-ph].

[16] O. J. P. Eboli, G. C. Marques, S. F. Novaes and A. A. Natale, Phys. Lett. B 197, 269 (1987).

[17] E. W. N. Glover and J. J. van der Bij, Nucl. Phys. B 309, 282 (1988); D. A. Dicus, C. Kao and S. S. D. Willenbrock, Phys. Lett. B 203, 457 (1988); T. Plehn, M. Spira and P. M. Zerwas, Nucl. Phys. B 479, 46 (1996) [Erratum-ibid. B 531, 655 (1998)].

[18] A. Djouadi, W. Kilian, M. Muhlleitner and P. M. Zerwas, Eur. Phys. J. C 10, 45 (1999).

[19] V. D. Barger, T. Han and R. J. N. Phillips, Phys. Rev. D 38, 2766 (1988).

[20] W. Y. Keung, Mod. Phys. Lett. A 2, 765 (1987); D. A. Dicus, K. J. Kallianpur and S. S. D. Willenbrock, Phys. Lett. B 200, 187 (1988).

[21] U. Baur, T. Plehn and D. L. Rainwater, Phys. Rev. Lett. 89, 151801 (2002), Phys. Rev. D 67, 033003 (2003), Phys. Rev. D 69, 053004 (2004); F. Gianotti et al., Eur. Phys. J. C 39, 293 (2005).

[22] G. J. Gounaris, D. Schildknecht and F. M. Renard, Phys. Lett. B 83, 191 (1979); A. Djouadi, H. E. Haber and P. M. Zerwas, Phys. Lett. B 375, 203 (1996); V.A. Ilyn et al., Phys. Rev. D 54, 6717 (1996).

[23] V. D. Barger and T. Han, Mod. Phys. Lett. A 5, 667 (1990).

[24] J. i. Kamoshita, Y. Okada, M. Tanaka and I. Watanabe, in Proceedings of Workshop on Physics and Experiments with Linear Colliders (Morioka-Appi, Iwate, Japan, 1995), p418; A. Djouadi, W. Kilian, M. Muhlleitner and P. M. Zerwas, Eur. Phys. J. C 10, 45 (1999); C. Castanier, P. Gay, P. Lutz and J. Orloff, hep-ex/0101028.

[25] M. Battaglia, E. Boos and W.-M. Yao, in Proceedings of the APS/DPF/DPB Summer Study on the Future of Particle Physics (Snowmass 2001); Y. Yasui, S. Kanemura, S. Kiyoura, K. Odagiri, Y. Okada, E. Senaha and S. Yamashita, arXiv:hep-ph/0211047.

[26] U. Baur, T. Plehn and D. L. Rainwater, Phys. Rev. D 68, 033001 (2003).

[27] U. Baur, Phys. Rev. D 80, 013012 (2009).

[28] M. F. Giannelli, arXiv:0901.4895 [hep-ex]; Y. Takubo, arXiv:0907.0524 [hep-ph]; J. Tian, K. Fujii and Y. Gao, arXiv:1008.0921 [hep-ex].

[29] G. V. Jikia, Nucl. Phys. B 412, 57 (1994); R. Belusevic and G. Jikia, Phys. Rev. D 70, 073017 (2004).

[30] E. Asakawa, D. Harada, S. Kanemura, Y. Okada and K. Tsumura, arXiv:0902.2458 [hep-ph]. 
[31] T. Takahashi et al., arXiv:0902.3377 [hep-ex]; K. Fujii, K. Ikematsu, S. Kanemura, Y. Kurihara, N. Maeda and T. Takahashi, arXiv:1008.0907 [hep-ph].

[32] H. M. Georgi, S. L. Glashow, M. E. Machacek and D. V. Nanopoulos, Phys. Rev. Lett. 40, 692 (1978).

[33] T. Barklow, in Reseach Directions for the Decade: Snowmass '90, edited by E. Berger (World Scientific, River Edge, NJ, 1992), p440; J. F. Gunion and H. E. Haber, in Reseach Directions for the Decade: Snowmass '90, edited by E. Berger (World Scientific, River Edge, NJ, 1992), p469, Phys. Rev. D 48, 5109 (1993).

[34] F. Boudjema and E. Chopin, Z. Phys. C 73, 85 (1996); V. Barger, T. Han, P. Langacker, B. McElrath and P. Zerwas, Phys. Rev. D 67, 115001 (2003).

[35] J. Pumplin, D. R. Stump, J. Huston, H. L. Lai, P. M. Nadolsky and W. K. Tung, JHEP 0207, $012(2002)$.

[36] T. Hahn and M. Perez-Victoria, Comput. Phys. Commun. 118, 153 (1999).

[37] S. Dawson, S. Dittmaier and M. Spira, Phys. Rev. D 58, 115012 (1998).

[38] I. F. Ginzburg, G. L. Kotkin, S. L. Panfil, V. G. Serbo and V. I. Telnov, Nucl. Instrum. Meth. A 219, 5 (1984).

[39] A. Pukhov et al., arXiv:hep-ph/9908288.

[40] For the review, J. F. Gunion, H. E. Haber, G. Kane and S. Dawson, The Higgs Hunter's Guide (Frontiers in Physics series, Addison-Wesley, Reading, MA, 1990)

[41] For reviews; H. E. Haber and G. L. Kane, Phys. Rept. 117, 75 (1985); S. P. Martin, arXiv:hep-ph/9709356.

[42] C. T. Hill, Phys. Lett. B 266, 419 (1991); Phys. Lett. B 345, 483 (1995).

[43] A. Zee, Phys. Lett. B 93, 389 (1980) [Erratum-ibid. B 95, 461 (1980)].

[44] N. Turok and J. Zadrozny, Phys. Rev. Lett. 65 (1990) 2331; N. Turok and J. Zadrozny, Nucl. Phys. B 358 (1991) 471; A. I. Bochkarev, S. V. Kuzmin and M. E. Shaposhnikov, Phys. Rev. D 43 (1991) 369; A. E. Nelson, D. B. Kaplan and A. G. Cohen, Nucl. Phys. B 373 (1992) 453; A. I. Bochkarev, S. V. Kuzmin and M. E. Shaposhnikov, Phys. Lett. B 244 (1990) 275; N. Turok and J. Zadrozny, Nucl. Phys. B 369 (1992) 729; K. Funakubo, A. Kakuto and K. Takenaga, Prog. Theor. Phys. 91, 341 (1994); A. T. Davies, C. D. froggatt, G. Jenkins and R. G. Moorhouse, Phys. Lett. B 336, 464 (1994). J. M. Cline and P. A. Lemieux, Phys. Rev. D 55, 3873 (1997).

[45] B. W. Lee, C. Quigg and H. B. Thacker, Phys. Rev. D 16, 1519 (1977).

[46] H. Huffel and G. Pocsik, Z. Phys. C 8, 13 (1981); J. Maalampi, J. Sirkka and I. Vilja, Phys. Lett. B 265, 371 (1991); S. Kanemura, T. Kubota and E. Takasugi, Phys. Lett. B 313, 155 (1993); A. G. Akeroyd, A. Arhrib and E. M. Naimi, Phys. Lett. B 490, 119 (2000); I. F. Ginzburg and I. P. Ivanov, Phys. Rev. D 72, 115010 (2005).

[47] N. G. Deshpande and E. Ma, Phys. Rev. D 18, 2574 (1978); M. Sher, Phys. Rept. 179, 273 (1989).

[48] S. Nie and M. Sher, Phys. Lett. B 449, 89 (1999); S. Kanemura, T. Kasai and Y. Okada, Phys. Lett. B 471, 182 (1999). 
[49] J. F. Gunion and H. E. Haber, Phys. Rev. D 67, 075019 (2003).

[50] V. D. Barger, J. L. Hewett and R. J. N. Phillips, Phys. Rev. D 41, 3421 (1990); Y. Grossman, Nucl. Phys. B 426, 355 (1994).

[51] M. Aoki, S. Kanemura, K. Tsumura and K. Yagyu, Phys. Rev. D 80, 015017 (2009); V. Barger, H. E. Logan and G. Shaughnessy, Phys. Rev. D 79, 115018 (2009); S. Su and B. Thomas, Phys. Rev. D 79, 095014 (2009).

[52] T. G. Rizzo, Phys. Rev. D 38, 820 (1988); W. S. Hou and R. S. Willey, Phys. Lett. B 202, 591 (1988); C. Q. Geng and J. N. Ng, Phys. Rev. D 38, 2857 (1988) [Erratum-ibid. D 41, 1715 (1990)] [Phys. Rev. D 41, 1715 (1990)]; B. Grinstein, R. P. Springer and M. B. Wise, Nucl. Phys. B 339, 269 (1990).

[53] H. E. Haber and A. Pomarol, Phys. Lett. B 302, 435 (1993); A. Pomarol and R. Vega, Nucl. Phys. B 413, 3 (1994).

[54] A. Arhrib, R. Benbrik, C. H. Chen, R. Guedes and R. Santos, JHEP 0908, 035 (2009).

[55] C. S. Kim, K. Y. Lee and J. H. Song, Phys. Rev. D 64, 015009 (2001).

[56] A. Arhrib, R. Benbrik and C. W. Chiang, Phys. Rev. D 77, 115013 (2008)G R. N. Hodgkinson, D. Lopez-Val and J. Sola, Phys. Lett. B 673, 47 (2009); D. Lopez-Val and J. Sola, Phys. Rev. D 81, 033003 (2010).

[57] F. Cornet and W. Hollik, Phys. Lett. B 669, 58 (2008).

[58] E. Asakawa, D. Harada, S. Kanemura, Y. Okada and K. Tsumura, Phys. Lett. B 672, 354 (2009).

[59] A. Arhrib, R. Benbrik, C. H. Chen and R. Santos, Phys. Rev. D 80, 015010 (2009).

[60] For the review, Leptoquarks by S. Rolli and M. Tanabashi in K. Nakamura et al. [Particle Data Group], J. Phys. G 37, 075021 (2010).

[61] V. M. Abazov et al. [D0 Collaboration], Phys. Rev. D 71, 071104 (2005).

[62] V. M. Abazov et al. [D0 Collaboration], Phys. Lett. B 671, 224 (2009).

[63] V. M. Abazov et al. [D0 Collaboration], Phys. Rev. Lett. 99, 061801 (2007).

[64] K. Nakamura et al. [Particle Data Group], J. Phys. G 37, 075021 (2010); C. Amsler et al. [Particle Data Group], Phys. Lett. B 667, 1 (2008).

[65] A. Lister [CDF Collaboration], arXiv:0810.3349 [hep-ex], T. Aaltonen et al. [CDF Collaboration], Phys. Rev. Lett. 104, 091801 (2010).

[66] J. Erler and P. Langacker, Phys. Rev. Lett. 105, 031801 (2010).

[67] M. Hashimoto, Phys. Rev. D 81, 075023 (2010).

[68] T. Aaltonen et al. [CDF and D0 Collaboration], arXiv:1005.3216 [hep-ex].

[69] T. Appelquist, H. C. Cheng and B. A. Dobrescu, Phys. Rev. D 64, 035002 (2001).

[70] N. Arkani-Hamed, A. G. Cohen, E. Katz and A. E. Nelson, JHEP 0207, 034 (2002); N. ArkaniHamed, A. G. Cohen, E. Katz, A. E. Nelson, T. Gregoire and J. G. Wacker, JHEP 0208, 021 (2002). For the Higgs boson pair production process, see L. Wang, W. Wang, J. M. Yang and H. Zhang, Phys. Rev. D 76, 017702 (2007); L. Wang and J. M. Yang, Phys. Rev. D 77, 015020 (2008); X. F. Han, L. Wang and J. M. Yang, Nucl. Phys. B 825, 222 (2010).

[71] B. A. Dobrescu and C. T. Hill, Phys. Rev. Lett. 81, 2634 (1998). 
[72] G. Cacciapaglia, A. Deandrea, D. Harada and Y. Okada, arXiv:1007.2933 [hep-ph].

[73] G. Passarino and M. J. G. Veltman, Nucl. Phys. B 160, 151 (1979). 